Preprint typeset in JHEP style. - HYPER VERSION

CERN-TH/2001-071

UCCHEP/16-01

\title{
Prototype Graphs for Radiative Corrections to Polarized Chargino or Neutralino Production in Electron-Positron Annihilation
}

\author{
M.A. Diaz \\ Departamento de Fisica, Pontificia Universidad Católica de Chile, Santiago \\ 690441, Chile. \\ D.A. Ross * \\ Division Théorique, CERN, 1211 Geneva 23, Switzerland
}

\begin{abstract}
We present the contributions from all types of one-loop corrections to the scattering amplitude for the pair production of polarized charginos or neutralinos from polarized electron-positron annihilation. The contributions are classified in terms of "prototypes" distinguished by the number of particles inside the loops and their spins. The results are quoted in terms of the Veltman-Passarino functions in terms of general couplings and internal masses. The results can therefore be applied to any supersymmetric extension of the Standard Model or indeed to any polarized fermion pair production process in electron-positron annihilation.
\end{abstract}

KEYWORDS: chargino, neutralino, supersymmetry, polarized.

${ }^{*}$ On leave of absence from: Department of Physics and Astronomy, University of Southampton, Southampton SO17 1BJ, U.K. 


\section{Contents}

1. Introduction 1

2. Generalized polarized scattering amplitudes 3

3. Self-energy and vertex corrections

3.1 Self-energy insertions: 6

3.2 Triangle graphs: 7

4. Box graphs 8

5. Conclusions 8

\section{Introduction}

The results from the last days of the running of LEP, which suggest that the mass of the Higgs scalar is around $115 \mathrm{GeV}$, has further enhanced the expectation that supersymmetry is realized in Nature [1, 2]. A Supersymmetric extension of the Standard Model is the only known scenario which can stabilise the effective potential whilst maintaining the experimental limits on the gauge-boson masses [3], as well as providing the possibility for a scenario of Grand Unification with a natural heirachy.

Nevertheless, a confirmation of supersymmetry can only result from the experimental identification of supersymmetric partners. The cleanest signal comes from the fermionic superpartners of the gauge bosons and Higgs bosons, the charginos and neutralinos. Regrettably LEP produced no evidence whatsoever for the existence of such particles and their existence is expected to be confirmed or negated by the next generation of hadron colliders. Notwithstanding this, the cleanest signal is still expected from electron-positron colliders and as such a possible future linear collider has an important role to play in the identification and study of fermionic superpartners.

The production cross-section for these superpartners is sensitive to the parameters of the supersymmetry extension. Conversely, a study of the production crosssection can be used to deduce information about the supersymmetry parameters. As has been shown by Choi. et. al. [⿴], even more information can be gleaned from the study of cross-sections in which both the initial electron and positron beams are polarized and the spin polarization of the final state fermion is also measured. 
The polarized cross-section was studied in detail at the tree-level in [四]. It is known, however, [5, 6] that at least for certain regions of parameter space, the higher order corrections to the production cross-section can be substantial and they are also sensitive to supersymmetry breaking parameters such as the squark masses, which do not enter into the tree-level calculation.

Recently Blank and Hollick [7] have reported on a complete calculation of the higher order corrections to the forward-backward asymmetry in the case of polarized electron and positron beams, but did not consider the polarization of the final state fermions.

Reports of numerical calculations for higher order corrections are undoubtedly very useful, but in the case of supersymmetric theories they inevitably refer to a particular set of parameters and need to be entirely repeated should one wish to calculate the effect of higher order corrections in a different part of parameter space.

For this reason we present in this paper the contributions to the polarized scattering amplitudes from all the one-loop prototype diagrams that can occur in a supersymmetric extension of the Standard Model. A prototype diagram is defined by the spins of the internal particles of the loop and we present the results in terms of the Veltman-Passarino functions [8] for completely general values of the couplings and masses of the internal particles. The formulae presented in this paper can be applied to polarized chargino or neutralino production in electron-positron annihilation, or indeed to any process in which a pair of massive fermions is produced and their polarization measured.

Although the total correction to the cross-section is inevitably gauge invariant, the contribution from individual graphs is not, and we work consistently in the 't Hooft-Feynman gauge, in which a gauge boson with momentum $k$ and mass $M$ has the propagator

$$
\frac{-i g^{\mu \nu}}{\left(k^{2}-M^{2}\right)} .
$$

Some of the Veltman-Passarino functions are ultraviolet divergent. It is assumed that these divergences have been regulated using dimensional regularization. On the other hand the renormalization scheme is, in general, unspecified and is reflected in the precise value assigned to the finite parts of the ultraviolet divergent PassarinoVeltman functions. Mass renormalizations of self-energy insertions are physical (i.e. the renormalization has been affected on mass-shell) and physical wavefunction renormalization has been carried out for the external particles.

The mass of the electron is neglected throughout.

The paper is organized as follows: In section 2 we discuss the general formalism for the calculation of the polarized scattering amplitudes in terms of a set of coefficient functions $\mathcal{Q}_{L(R) i, j}$, which are a generalization of the coefficient functions used in [4] for the tree-level calculation. These coefficient functions multiply matrix elements of various Dirac-matrix structures, some of which have been relegated to Appendix A. 
In section 3, we discuss the contribution to these coefficient functions from self-energy and vertex-correction graphs in terms of the more familiar language of form-factors. The relations between the form-factors and the coefficient functions is given. The detailed contributions from the various prototype graphs to the self-energies are given in Appendix B, to the vertex corrections in Appendix C, and to the box-diagrams, which must be expressed directly in terms of the coefficient functions, in Appendix D. In section 4 we present our conclusions.

\section{Generalized polarized scattering amplitudes}

In this section we restrict our discussion to charginos, although it can be equally applied to neutralino production.

We write the scattering amplitude for an electron with helicity $\alpha=R, L$ and momentum $p_{1}$, and a positron of opposite helicity and momentum $p_{2}$ into a (positively charged) chargino of mass $m_{\chi_{b}}$ with momentum $k_{2}$ and helicity $\lambda_{2}$ and a (negatively charged) anti-chargino of mass $m_{\chi_{a}}$, momentum $k_{1}$ and helicity $\lambda_{1}$ as

$$
\mathcal{A}_{\lambda_{2}, \lambda_{1}}^{\alpha}=\frac{2}{s} L_{\alpha}^{\mu} Q_{\mu \alpha}^{i}\left\langle k_{2}, \lambda_{2}\left|\Gamma^{i}\right| k_{1}, \lambda_{1}\right\rangle . .
$$

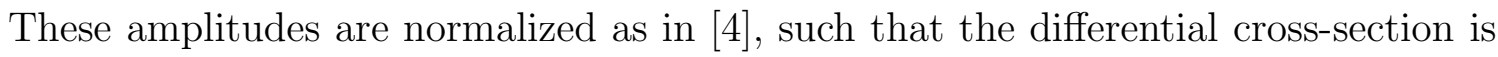
given by

$$
\frac{d \sigma\left(\alpha, \lambda_{2}, \lambda_{1}\right)}{d \cos \theta}=\frac{\lambda^{1 / 2}\left(s, m_{\chi_{a}}^{2}, m_{\chi_{b}}^{2}\right)}{128 \pi s}\left|\mathcal{A}_{\lambda_{2}, \lambda_{1}}^{\alpha}\right|^{2},
$$

where

$$
\lambda(x, y, z) \equiv x^{2}+y^{2}+z^{2}-2 x y-2 x z-2 y z
$$

The contribution from any Feynman graph to such an amplitude can always be expressed in this form by making a suitable Fierz transformation where necessary. Here $L_{R(L)}^{\mu}$ is the leptonic matrix element

$$
L_{R(L)}^{\mu}=\bar{v}\left(p_{2}\right) \gamma^{\mu} \frac{\left(1 \pm \gamma^{5}\right)}{2} u\left(p_{1}\right) .
$$

Since the leptons are considered to be massless these two are the only possible structures for the lepton factor. On the other hand the chargino factor is the sum of matrix elements of five possible $\gamma$-matrix structures $\Gamma_{i}, i=1 \cdots 5$ are given by

$$
\begin{aligned}
& \Gamma^{1}=\frac{\left(1+\gamma^{5}\right)}{2} \\
& \Gamma^{2}=\frac{\left(1-\gamma^{5}\right)}{2} \\
& \Gamma^{3}=\gamma^{\nu} \frac{\left(1+\gamma^{5}\right)}{2} \\
& \Gamma^{4}=\gamma^{\nu} \frac{\left(1-\gamma^{5}\right)}{2} \\
& \Gamma^{5}=-i \sigma^{\nu \rho}
\end{aligned}
$$


The coefficients $Q_{\mu \alpha}^{i}$ are tensors which can be reduced to the following structures, in terms of scalar quantities $\mathcal{Q}_{L(R) i, j}, j=1,2$, as follows

$$
\begin{aligned}
& Q_{L(R) 1}^{\mu}=\mathcal{Q}_{L(R) 1} k_{-}^{\mu} \\
& Q_{L(R) 2}^{\mu}=\mathcal{Q}_{L(R) 2} k_{-}^{\nu} \\
& Q_{L(R) 3}^{\mu \nu}=\mathcal{Q}_{L(R) 3,1} g^{\mu \nu}+\mathcal{Q}_{L(R) 3,2} k_{-}^{\mu} p^{\nu} \\
& Q_{L(R) 4}^{\mu \nu}=\mathcal{Q}_{L(R) 4,1} g^{\mu \nu}+\mathcal{Q}_{L(R) 4,2} k_{-}^{\mu} p^{\nu} \\
& Q_{L(R) 5}^{\mu \nu \rho}=\mathcal{Q}_{L(R) 5,1} g^{\mu \nu} p^{\rho}-i \mathcal{Q}_{L(R) 5,2} \epsilon^{\mu \nu \rho \tau} p_{\tau},
\end{aligned}
$$

where $k_{-}^{\mu}=\left(k_{1}^{\mu}-k_{2}^{\mu}\right)$ and $p^{\mu}=\left(p_{1}^{\mu}-p_{2}^{\mu}\right)$. Any other structure can be expressed in terms of the above quantities, by exploiting the fact that the leptonic current is conserved and that the matrix elements of $\Gamma^{i}$ are taken between on-shell chargino states. We note here that at the tree level, only $\mathcal{Q}_{L(R) 3,1}$ and $\mathcal{Q}_{L(R) 4,1}$ are non-zero. Furthermore $\mathcal{Q}_{L(R) 3,2}, \mathcal{Q}_{L(R) 4,2}, \mathcal{Q}_{L(R) 5,1}$ and $\mathcal{Q}_{L(R) 5,2}$ do not occur in self-energy or vertex correction graphs, but only arise when boxes are taken into consideration.

We express the contributions to the scattering amplitude multiplying the scalar quantities $\mathcal{Q}_{L(R) i, j}$, using the following notation:

$k$ is the 3 -momentum of the $\chi$ in the C.M. frame and $\theta$ is the scattering angle in the C.M. frame, i.e. the angle between the incident electron and the final-state $\chi^{-}$. We define further:

$$
\begin{aligned}
& f_{+}=\sqrt{\left(s-m_{\chi_{a}}^{2}-m_{\chi_{b}}^{2}\right)+2 k \sqrt{s}} \\
& f_{-}=\sqrt{\left(s-m_{\chi_{a}}^{2}-m_{\chi_{b}}^{2}\right)-2 k \sqrt{s}}
\end{aligned}
$$

The scattering amplitude contributions from each of the $\mathcal{Q}_{L(R) i, j}$ are given by:

$$
\begin{gathered}
-\mathcal{Q}_{L(R) 1}\left\{\frac{2 \sqrt{2} k \sin \theta}{\sqrt{s}}\left(f_{-} \delta_{\lambda_{2}+} \delta_{\lambda_{1}+}+f_{+} \delta_{\lambda_{2}-} \delta_{\lambda_{1}-}\right)\right\} \\
\mathcal{Q}_{L(R) 2}\left\{\frac{2 \sqrt{2} k \sin \theta}{\sqrt{s}}\left(f_{+} \delta_{\lambda_{2}+} \delta_{\lambda_{1}+}+f_{-} \delta_{\lambda_{2}-} \delta_{\lambda_{1}-}\right)\right\} \\
-\mathcal{Q}_{R, 3,1}\left\{\frac{2 k \sin \theta}{\sqrt{s}}\left(a_{1}\left(R, \lambda_{2}, \lambda_{1}\right)-a_{2}\left(R, \lambda_{2}, \lambda_{1}\right)\right)-2 a_{4}\left(\left(R, \lambda_{2}, \lambda_{1}\right)\right\},\right. \\
-\mathcal{Q}_{L, 3,1}\left\{\frac{2 k \sin \theta}{\sqrt{s}}\left(a_{1}\left(R, \lambda_{2}, \lambda_{1}\right)-a_{2}\left(R, \lambda_{2}, \lambda_{1}\right)\right)+2 a_{4}\left(\left(R, \lambda_{2}, \lambda_{1}\right)\right\}\right. \\
-\mathcal{Q}_{R, 4,1}\left\{\frac{2 k \sin \theta}{\sqrt{s}}\left(a_{1}\left(L, \lambda_{2}, \lambda_{1}\right)-a_{2}\left(L, \lambda_{2}, \lambda_{1}\right)\right)-2 a_{4}\left(\left(L, \lambda_{2}, \lambda_{1}\right)\right\}\right. \\
-\mathcal{Q}_{L, 4,1}\left\{\frac{2 k \sin \theta}{\sqrt{s}} \tilde{a}_{3}\left(R, \lambda_{2}, \lambda_{1}\right)\right\} \\
\sqrt{s}\left(a_{1}\left(L, \lambda_{2}, \lambda_{1}\right)-a_{2}\left(L, \lambda_{2}, \lambda_{1}\right)\right)+2 a_{4}\left(\left(L, \lambda_{2}, \lambda_{1}\right)\right\}
\end{gathered}
$$




$$
\begin{gathered}
-\mathcal{Q}_{R(L), 4,2}\left\{\frac{4 k \sin \theta}{\sqrt{s}} \tilde{a}_{3}\left(L, \lambda_{2}, \lambda_{1}\right)\right\}, \\
\mathcal{Q}_{R, 5,1}\left\{\sqrt { 2 } ( f _ { + } - f _ { - } ) \left[\sin \theta \delta_{\lambda_{2} \lambda_{1}}\right.\right. \\
\left.\left.+\frac{\left(m_{\chi_{a}}-m_{\chi_{b}}\right)}{\sqrt{s}}\left(\cos \theta \operatorname{sgn}\left(\lambda_{1}\right)+1\right) \delta_{\lambda_{2},-\lambda_{1}}\right]\right\}, \\
-\mathcal{Q}_{L, 5,1}\left\{\sqrt { 2 } ( f _ { + } - f _ { - } ) \left[\sin \theta \delta_{\lambda_{2} \lambda_{1}}\right.\right. \\
\left.\left.+\frac{\left(m_{\chi_{a}}-m_{\chi_{b}}\right)}{\sqrt{s}}\left(\cos \theta \operatorname{sgn}\left(\lambda_{1}\right)-1\right) \delta_{\lambda_{2},-\lambda_{1}}\right]\right\}, \\
-\mathcal{Q}_{R, 5,2}\left\{2 \sqrt { 2 } ( f _ { + } + f _ { - } ) \left[\sin \theta \delta_{\lambda_{2} \lambda_{1}} \operatorname{sgn}\left(\lambda_{1}\right)\right.\right. \\
\left.\left.-\frac{\left(m_{\chi_{a}}+m_{\chi_{b}}\right)}{\sqrt{s}}\left(\cos \theta+\operatorname{sgn}\left(\lambda_{1}\right)\right) \delta_{\lambda_{2},-\lambda_{1}}\right]\right\}, \\
\mathcal{Q}_{L, 5,2}\left\{2 \sqrt { 2 } ( f _ { + } + f _ { - } ) \left[\sin \theta \delta_{\lambda_{2} \lambda_{1}} \operatorname{sgn}\left(\lambda_{1}\right)\right.\right. \\
\left.\left.-\frac{\left(m_{\chi_{a}}+m_{\chi_{b}}\right)}{\sqrt{s}}\left(\cos \theta-\operatorname{sgn}\left(\lambda_{1}\right)\right) \delta_{\lambda_{2},-\lambda_{1}}\right]\right\} .
\end{gathered}
$$

The amplitudes, $a_{i}\left(\alpha, \lambda_{2}, \lambda_{1}\right),,(i=1 \cdots 4)$ and $\tilde{a}_{3}\left(\left(\alpha, \lambda_{2}, \lambda_{1}\right)\right.$ are given in Appendix A.

\section{Self-energy and vertex corrections}

The coefficient functions, $\mathcal{Q}_{L(R) i, j}$, which acquire contributions from self-energy insertions and triangle graphs are best treated in terms of form-factors, which replace point-like vertices in the tree-level contributions that arise either from a graph involving an exchange in the $s$-channel of a vector boson, or the $t$-channel exchange of a scalar particle ( a sneutrino in the case of chargino pair production from electronpositron annihilation).

We write the general vertex for a vector boson coupling to an anti-fermion momentum fermion with (on-shell) momenta $k_{1}$ and $k_{2}$ respectively, as

$$
F_{0}^{+} \gamma^{\mu} \frac{\left(1+\gamma^{5}\right)}{2}+F_{0}^{-} \gamma^{\mu} \frac{\left(1-\gamma^{5}\right)}{2}+F_{k}^{+} k_{-}^{\mu} \frac{\left(1+\gamma^{5}\right)}{2}+F_{k}^{-} k_{-}^{\mu} \frac{\left(1-\gamma^{5}\right)}{2} .
$$

Note that the form-factors $F_{k}^{+}, F_{k}^{-}$are absent at tree-level. 
The Yukawa coupling of fermions to a scalar particle is given by

$$
F_{\tilde{\nu}}^{ \pm} \frac{\left(1 \pm \gamma^{5}\right)}{2}
$$

for right-(left-) handed incoming fermions respectively.

Thus the contributions to the coefficient functions from the exchange of a vector boson of mass $M_{V}$ in the $s$-channel are:

$$
\begin{aligned}
\Delta \mathcal{Q}_{L(R) 3,1} & =g_{L(R)} \frac{s}{\left(s-M_{V}^{2}\right)} F_{0(V)}^{+}, \\
\Delta \mathcal{Q}_{L(R) 4,1} & =g_{L(R)} \frac{s}{\left(s-M_{V}^{2}\right)} F_{0(V)}^{-}, \\
\Delta \mathcal{Q}_{L(R) 1} & =g_{L(R)} \frac{s}{\left(s-M_{V}^{2}\right)} F_{k(V)}^{+}, \\
\Delta \mathcal{Q}_{L(R) 2} & =g_{L(R)} \frac{s}{\left(s-M_{V}^{2}\right)} F_{k(V)}^{-},
\end{aligned}
$$

where $g_{L(R)}$ is the coupling of the left-(right-)handed electrons to the vector boson.

Upon performing a Fierz transformation, the contributions to the coefficient functions from the exchange of a scalar particle of mass $m_{\tilde{\nu}}$ in the $t$-channel, is given by

$$
\Delta \mathcal{Q}_{L 4,1}=-\frac{1}{2} \frac{s}{\left(t-m_{\tilde{\nu}}^{2}\right)} F_{\tilde{\nu}}^{-}\left(F_{\tilde{\nu}}^{-}\right)^{\dagger},
$$

for a scalar particle that couples to left-handed incoming electrons and

$$
\Delta \mathcal{Q}_{R 3,1}=-\frac{1}{2} \frac{s}{\left(t-m_{\tilde{\nu}}^{2}\right)} F_{\tilde{\nu}}^{+}\left(F_{\tilde{\nu}}^{+}\right)^{\dagger},
$$

for a scalar particle that couples to right-handed incoming electrons.

Note that $F_{\tilde{\nu}}^{ \pm}$are the form-factors for an incoming electron and outgoing antichargino or anti-neutralino with helicities \pm , whereas $\left(F_{\tilde{\nu}}^{ \pm}\right)^{\dagger}$ are the form-factors for an incoming positron and outgoing chargino or neutralino with helicities \pm ,

\subsection{Self-energy insertions:}

The contribution to the form-factors $F_{\tilde{\nu}}^{ \pm}$from the self-energy $\Sigma_{\tilde{\nu}}(t)$ of the scalar particle exchanged in the $t$-channel is given by

$$
\Delta F_{\tilde{\nu}}^{ \pm}=\frac{\left(\Sigma_{\tilde{\nu}}(t)-\Sigma_{\tilde{\nu}}\left(m_{\tilde{\nu}}^{2}\right)\right)}{\left(t-m_{\tilde{\nu}}^{2}\right)} F_{\tilde{\nu}}^{ \pm} .
$$

For the exchange of a gauge-boson in the $s$-channel, we need to account for possible mixing at the one-loop level between a gauge-boson $V$ and a gauge-boson $V^{\prime}$. The transverse part of the self-energy can be written in general as $\Sigma_{V V^{\prime}}(s)$, and the contribution to the form-factors $F_{0(V)}^{ \pm}$is given by 


$$
\Delta F_{0(V)}^{ \pm}=\frac{\left(\Sigma_{V V^{\prime}}(s)-\Sigma_{V V^{\prime}}\left(M_{V^{\prime}}^{2}\right)\right)}{\left(s-M_{V^{\prime}}^{2}\right)} F_{0\left(V^{\prime}\right)}^{ \pm} .
$$

The contribution to the form-factors from the self-energy of the charginos (or neutralinos) is more complicated owing to different one-loop contributions to leftand right-handed components and to the mixing between the different species of charginos (neutralinos). The was discussed in detail in [5]. Here we reproduce the main results.

The general self-energy of a chargino or neutralino of momentum $p$ can be written as

$$
\Sigma_{a b}(p)=\left(A_{a b}^{+}\left(p^{2}\right)+B_{a b}^{+}\left(p^{2}\right) \gamma \cdot p\right) \frac{\left(1+\gamma^{5}\right)}{2}+\left(A_{a b}^{-}\left(p^{2}\right)+B_{a b}^{-}\left(p^{2}\right) \gamma \cdot p\right) \frac{\left(1-\gamma^{5}\right)}{2}
$$

where the suffix $a b$ refers to an outgoing chargino (neutralino) of type $a$ and an incoming chargino (neutralino) of type $b$.

We denote $F^{ \pm a}$ to mean the form-factor involving a chargino or neutralino of type $a$ (with mass $m_{a}$ ).

The correction to the form-factors due to the self-energies of the external fermion is given by

$$
\begin{aligned}
\Delta F^{ \pm a} & =\frac{1}{2}\left\{ \pm \frac{\left(A_{a a}^{+}-A_{a a}^{-}\right)}{2 m_{a}}+B_{a a}^{ \pm}+m_{a}\left(A_{a a}^{+\prime}+A_{a a}^{-\prime}+m_{a}\left(B_{a a}^{+\prime}+B_{a a}^{-\prime}\right)\right)\right\} F^{ \pm a} \\
& +\sum_{b \neq a}\left\{\frac{m_{a} A_{a b}^{ \pm}+m_{b} A_{a b}^{\mp}+m_{a}^{2} B_{a b}^{ \pm}+m_{a} m_{b} B_{a b}^{\mp}}{\left(m_{a}^{2}-m_{b}^{2}\right)}\right\} F^{ \pm b}
\end{aligned}
$$

for the self-energy correction to a chargino (neutralino), and

$$
\begin{aligned}
\Delta F^{ \pm a} & =\frac{1}{2}\left\{ \pm \frac{\left(A_{a a}^{-}-A_{a a}^{+}\right)}{2 m_{a}}+B_{a a}^{ \pm}+m_{a}\left(A_{a a}^{+\prime}+A_{a a}^{-\prime}+m_{a}\left(B_{a a}^{+\prime}+B_{a a}^{-\prime}\right)\right)\right\} F^{ \pm a} \\
& +\sum_{b \neq a}\left\{\frac{m_{a} A_{b a}^{\mp}+m_{b} A_{b a}^{ \pm}+m_{a}^{2} B_{b a}^{ \pm}+m_{a} m_{b} B_{b a}^{\mp}}{\left(m_{a}^{2}-m_{b}^{2}\right)}\right\} F^{ \pm b}
\end{aligned}
$$

for the self-energy correction to an anti-chargino (anti-neutralino). The $/$ refers to the derivative w.r.t. $p^{2}$ and all the functions are taken at the point $p^{2}=m_{a}^{2}$.

The contributions from the various prototype diagrams to the self energies, $\Sigma_{\tilde{\nu}}, \Sigma_{V V^{\prime}}, \Sigma_{a b}$ are given in Appendix B.

\subsection{Triangle graphs:}

The contributions to the form-factors, $F_{0}^{ \pm}, F_{k}^{ \pm}, F_{\tilde{\nu}}^{ \pm}$from the different possible triangle graphs are given in Appendix C.

We note here that for a complete one-loop calcualtion, we also need to account

for the form-factor of the vertex between the intermediate gauge bosons and the 
incoming electron-positron pair. Since we neglect the masses of the leptons only the form-factors, $F_{0}^{ \pm}$are non-zero. The contributions to these are obtained form the vertex protoypes $5-8$ by setting $m_{\chi_{a}}, m_{\chi_{b}}=0$.

\section{Box graphs}

The contibutions to the coefficient functions $\mathcal{Q}_{L(R) i, j}$ from all the prototype box graphs are given in Appendix D. All box graphs contributing to the process under consideration can be expressed in terms of these prototypes or prototypes which can be obtained from them by the simple crossing relations $t \leftrightarrow u, m_{\chi_{a}} \leftrightarrow m_{\chi_{b}}$.

\section{Conclusions}

In this paper we have presented explicit expressions for the contributions from all prototypes of one-loop corrections in terms of the Veltman-Passarino functions. The results are given in terms of general couplings and internal masses, so that they can be used in any model for the determination of the scattering amplitudes of polarized fermion pair production.

The results are presented in two stages. Firstly, the helicity amplitudes are given in terms of a small number of coefficient functions, $\mathcal{Q}_{L(R) i, j}$, of various effective interaction structures. Secondly the contributions from all prototype Feynman diagrams to these coefficient functions are given.

There exist [8] relations between the Veltman-Passarino functions used in this paper, such that they can all be reduced to "scalar" types functions, $A 0, B 0, C 0$ and $D 0$. We have chosen not to implement these relations in the expressions presented here. With the exception of cases in which some of the internal particles masses vanish, the expressions tend be be considerably longer when only this minimal set of functions is used. Instead, it is probably more efficient to implement these relations at the point of numerical calculations. A FORTRAN library exists 10 for the numerical calculation of the Veltman-Passarino functions, but unfortunately these only calculate such functions with up to one power of the loop momentum in the numerator. We have written a set of FORTRAN subroutines which are to be used in conjunction with this library and can be used to calculate the Veltman-Passarino functions with up to three powers of loop momenta in the numerator (for the calculation addressed in this paper only two powers of loop momenta are required). These routines are available upon request as well as a set of subroutines that calculate the

contributions to the coefficient functions, $\mathcal{Q}_{L(R) i, j}$, from all the prototype diagrams considered in this paper. 


\section{Acknowledgments}

The authors wish to thank Steve King for useful conversations, assistance, and many helpful suggestions.

Many of the algebraic manipulations performed in this work were carried out using FORM [11].

This work was partially supported by CONICYT grant No: 1010974 .

\section{Appendix A}

The quantities $a_{i}\left(\alpha, \lambda_{2}, \lambda_{1}\right)$ used in eqs.(2.9-2.14) are given by

$$
a_{i}\left(\alpha, \lambda_{2}, \lambda_{1}\right)=R_{i}^{j} \tilde{a}_{j}\left(\alpha, \lambda_{2}, \lambda_{1}\right) .
$$

The $4 \times 4$ matrix, $R$, can be written in terms of the is the the matrix $3 \times 3$ matrix, $T$ as

$$
R=\frac{1}{s k^{2} \sin ^{2} \theta}\left(\begin{array}{ll}
T & \\
& -k^{2} \sin ^{2} \theta
\end{array}\right),
$$

where

$$
T=\left(\begin{array}{ccc}
-m_{\chi_{b}}^{2}-k^{2} \cos ^{2} \theta & \frac{1}{2} s_{--}-k^{2} \cos ^{2} \theta & \frac{k \cos \theta}{2 \sqrt{s}} s_{-+} \\
\frac{1}{2} s_{--}-k^{2} \cos ^{2} \theta & -m_{\chi_{a}}^{2}-k^{2} \cos ^{2} \theta & -\frac{k \cos \theta}{2 \sqrt{s}} s_{+-} \\
\frac{k \cos \theta}{2 \sqrt{s}} s_{-+} & -\frac{k \cos \theta}{2 \sqrt{s}} s_{+-} & -k^{2}
\end{array}\right),
$$

with $s_{--}=s-m_{\chi_{a}}^{2}-m_{\chi_{b}}^{2}, s_{+-}=s+m_{\chi_{a}}^{2}-m_{\chi_{b}}^{2}$, and $s_{-+}=s-m_{\chi_{a}}^{2}+m_{\chi_{b}}^{2}$. The quantities $\tilde{a}_{i}\left(\alpha, \lambda_{2}, \lambda_{1}\right)$ are given (in four vector notation, $i=1 \cdots 4$ ) by

$$
\begin{aligned}
& \tilde{a}(R,+,+)=\frac{1}{\sqrt{2}}\left(m_{\chi_{a}} f_{+}, m_{\chi_{b}} f_{-},\left(m_{\chi_{a}} f_{+}+m_{\chi_{b}} f_{-}\right) \cos \theta, 0\right) \\
& \tilde{a}(R,+,-)=\frac{1}{\sqrt{2}}\left(0,0, \sqrt{s} f_{+} \sin \theta,-\sqrt{s} f_{+}\right) \\
& \tilde{a}(R,-,+)=\frac{1}{\sqrt{2}}\left(0,0, \sqrt{s} f_{-} \sin \theta, \sqrt{s} f_{-}\right) \\
& \tilde{a}(R,-,-)=\frac{1}{\sqrt{2}}\left(m_{\chi_{a}} f_{-}, m_{\chi_{b}} f_{+},-\left(m_{\chi_{a}} f_{-}+m_{\chi_{b}} f_{+}\right) \cos \theta, 0\right) \\
& \tilde{a}(L,+,+)=\frac{1}{\sqrt{2}}\left(-m_{\chi_{a}} f_{-},-m_{\chi_{b}} f_{+},\left(m_{\chi_{a}} f_{-}+m_{\chi_{b}} f_{+}\right) \cos \theta, 0\right) \\
& \tilde{a}(L,+,-)=\frac{1}{\sqrt{2}}\left(0,0, \sqrt{s} f_{-} \sin \theta,-\sqrt{s} f_{-}\right) \\
& \tilde{a}(L,-,+)=\frac{1}{\sqrt{2}}\left(0,0, \sqrt{s} f_{+} \sin \theta, \sqrt{s} f_{+}\right) \\
& \tilde{a}(L,-,-)=\frac{1}{\sqrt{2}}\left(-m_{\chi_{a}} f_{+},-m_{\chi_{b}} f_{-},-\left(m_{\chi_{a}} f_{+}+m_{\chi_{b}} f_{-}\right) \cos \theta, 0\right)
\end{aligned}
$$

Note that the quantities $\tilde{a}_{3}\left(R(L)\left(\lambda_{2}, \lambda_{1}\right)\right.$ of eqs. (2.11, 2.14) are the third components of these vectors. 


\section{Appendix B}

\section{Prototype self-energy graphs}

The self-energy corrections in a general supersymmetric theory have been consid-

ered in detail in [9]. For completeness, and to conform with the notation used in this paper, we repeat the results for the various self-energy prototype diagrams in this Appendix, in terms of the Veltman-Passarino functions, $A 0, B 0, B 1, B 21, B 22$, defined as

$$
\begin{aligned}
& -i \int \frac{d^{4} l}{(2 \pi)^{4}} \frac{1}{\left(l^{2}-m_{1}^{2}\right)\left((l+v)^{2}-m_{2}^{2}\right)}=B 0 \\
& -i \int \frac{d^{4} l}{(2 \pi)^{4}} \frac{l^{\rho}}{\left(l^{2}-m_{1}^{2}\right)\left((l+v)^{2}-m_{2}^{2}\right)}=B 1 v^{\rho}, \\
& -i \int \frac{d^{4} l}{(2 \pi)^{4}} \frac{l^{2}}{\left(l^{2}-m_{1}^{2}\right)\left((l+v)^{2}-m_{2}^{2}\right)}=A 0\left(m_{2}^{2}\right)+m_{1}^{2} B 0 \\
& -i \int \frac{d^{4} l}{(2 \pi)^{4}} \frac{l^{\rho} l^{\sigma}}{\left(l^{2}-m_{1}^{2}\right)\left((l+v)^{2}-m_{2}^{2}\right)}=g^{\rho \sigma} B 22+B 21 v^{\rho} v^{\sigma} .
\end{aligned}
$$

\section{Scalar self-energy}

\section{SELF-ENERGY PROTOTYPE 1}

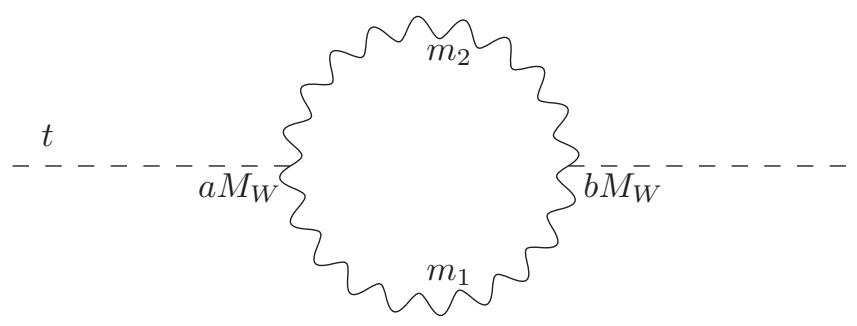

Figure 1: Self-energy prototype graph 1.

$$
16 \pi^{2} \Delta \Sigma_{\tilde{\nu}}(t)=4 a b M_{W}^{2}\left[B 0-\frac{1}{2}\right]
$$




\section{SELF-ENERGY PROTOTYPE 2}

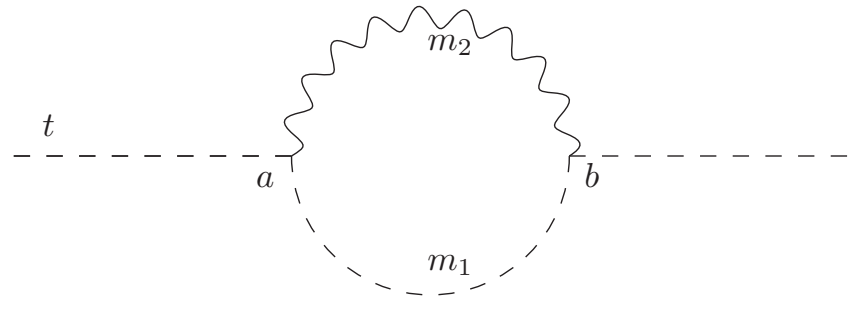

Figure 2: Self-energy prototype graph 2.

$$
16 \pi^{2} \Delta \Sigma_{\tilde{\nu}}(t)=-a b\left[\left(t+m_{1}^{2}\right) B 0-2 t B 1+A 0\right]
$$

\section{SELF-ENERGY PROTOTYPE 3}

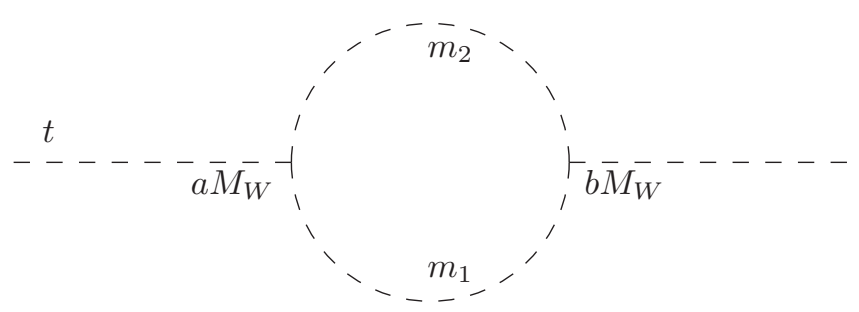

Figure 3: Self-energy prototype graph 3.

$$
16 \pi^{2} \Delta \Sigma_{\tilde{\nu}}(t)=a b M_{W}^{2} B 0
$$

\section{SELF-ENERGY PROTOTYPE 4}

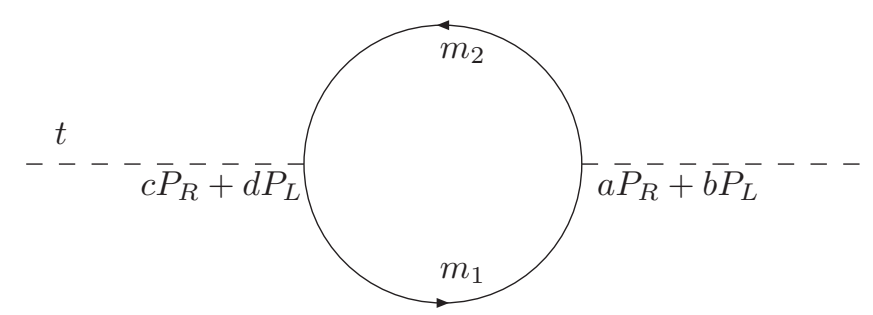

Figure 4: Self-energy prototype graph 4.

$$
16 \pi^{2} \Delta \Sigma_{\tilde{\nu}}(t)=-2(a d+b c)\left[A 0+m_{1}^{2} B_{0}+t B 1\right]-2(a c+b d) m_{1} m_{2} B 0
$$




\section{Vector self-energy}

\section{SELF-ENERGY PROTOTYPE 5}

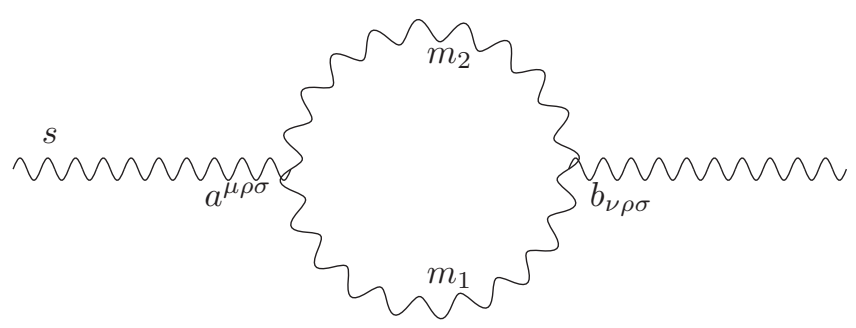

Figure 5: Self-energy prototype graph 5.

$a^{\mu \rho \sigma}$ and $b_{\nu \rho \sigma}$ are the usual triple gauge boson vertices with gauge couplings $a$ and $b$ respectively. The contribution from Faddeev-Popov ghosts has been added to this graph.

$$
16 \pi^{2} \Delta \Sigma_{V V \prime}(s)=-a b\left[8 B 22+2 A 0+\left(5 s+2 m_{1}^{2}\right) B 0+2 s B 1+\frac{2}{3} s\right]
$$

\section{SELF-ENERGY PROTOTYPE 6}

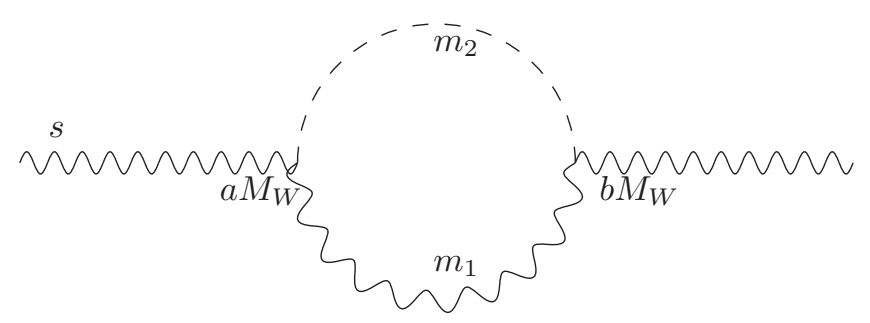

Figure 6: Self-energy prototype graph 6.

$$
16 \pi^{2} \Delta \Sigma_{V V \prime}(s)=a b M_{W}^{2} B 0
$$

\section{SELF-ENERGY PROTOTYPE 7}

$$
16 \pi^{2} \Delta \Sigma_{V V \prime}(s)=-4 a b B 22
$$




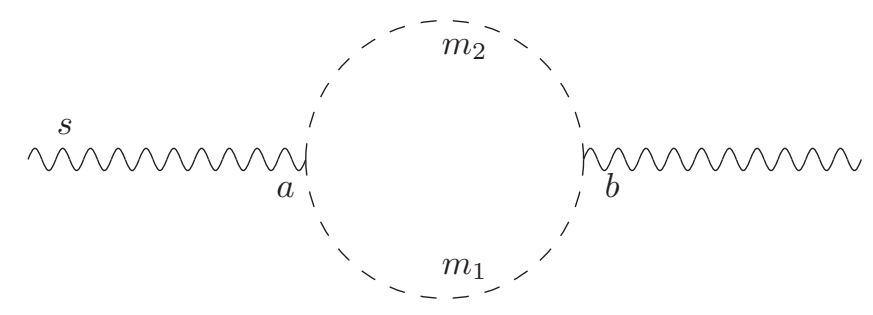

Figure 7: Self-energy prototype graph 7.

\section{SELF-ENERGY PROTOTYPE 8}

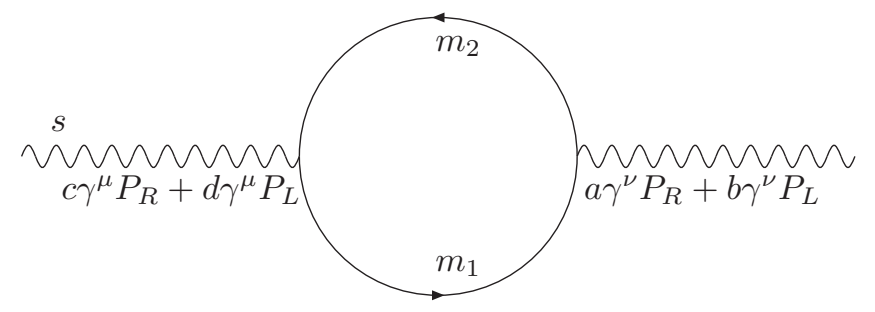

Figure 8: Self-energy prototype graph 8.

$16 \pi^{2} \Delta \Sigma_{V V^{\prime}}(s)=2(a d+b c) m_{1} m_{2} B 0-2(a c+b d)\left[A 0+m_{1}^{2} B 0+s B 1-2 B 22\right]$

\section{Fermion self-energy}

\section{SELF-ENERGY PROTOTYPE 9}

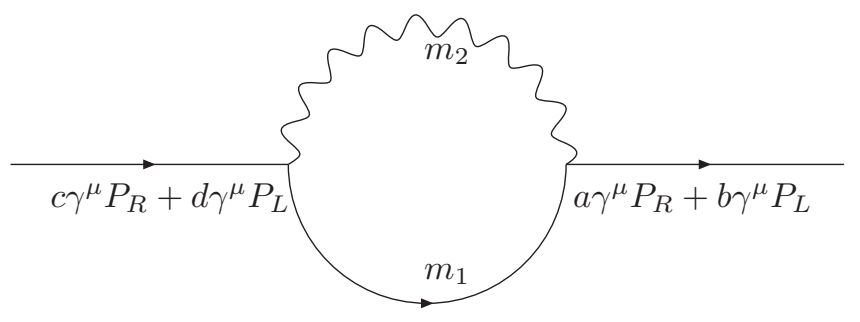

Figure 9: Self-energy prototype graph 9.

$$
16 \pi^{2} \Delta A^{+}=-4 b c m_{1}\left[B 0-\frac{1}{2}\right]
$$




$$
\begin{aligned}
16 \pi^{2} \Delta A^{-} & =-4 a d m_{1}\left[B 0-\frac{1}{2}\right] \\
16 \pi^{2} \Delta B^{+} & =-2 a c\left[B 1+\frac{1}{2}\right] \\
16 \pi^{2} \Delta B^{-} & =-2 b d\left[B 1+\frac{1}{2}\right]
\end{aligned}
$$

\section{SELF-ENERGY PROTOTYPE 10}

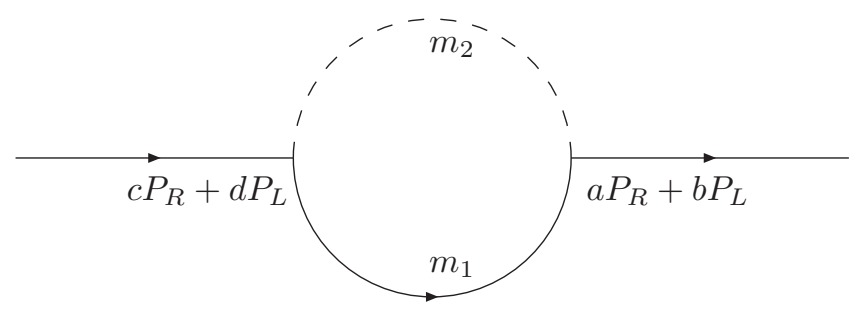

Figure 10: Self-energy prototype graph 10.

$$
\begin{aligned}
& 16 \pi^{2} \Delta A^{+}=a c m_{1} B 0 \\
& 16 \pi^{2} \Delta A^{+}=b d m_{1} B 0 \\
& 16 \pi^{2} \Delta B^{+}=-b c B 1 \\
& 16 \pi^{2} \Delta B^{-}=-a d B 1
\end{aligned}
$$

\section{Appendix C}

\section{Prototype triangle graphs}

In this Appendix we give the expressions for the prototype triangle diagrams, with couplings indicated in the diagrams, in terms of the Veltman-Passarino functions, $C 0, C 11, C 12, C 21, C 23, C 24$, These functions, with arguments $v_{1}^{2}, v_{2}^{2},\left(v_{1}+\right.$ $\left.v_{2}\right)^{2}, m_{1}^{2}, m_{2}^{2}, m_{3}^{2}$ are defined as

$$
\begin{aligned}
& -i \int \frac{d^{4} l}{(2 \pi)^{4}} \frac{1}{\left.\left(l^{2}-m_{1}^{2}\right)\left(\left(l+v_{1}\right)^{2}-m_{2}^{2}\right)\left(l+v_{1}+v_{2}\right)^{2}-m_{3}^{2}\right)}=C 0 \\
& -i \int \frac{d^{4} l}{(2 \pi)^{4}} \frac{l^{\rho}}{\left.\left(l^{2}-m_{1}^{2}\right)\left(\left(l+v_{1}\right)^{2}-m_{2}^{2}\right)\left(l+v_{1}+v_{2}\right)^{2}-m_{3}^{2}\right)}=C 11 v_{1}^{\rho}+C 12 v_{2}^{\rho} \\
& -i \int \frac{d^{4} l}{(2 \pi)^{4}} \frac{l^{2}}{\left.\left(l^{2}-m_{1}^{2}\right)\left(\left(l+v_{1}\right)^{2}-m_{2}^{2}\right)\left(l+v_{1}+v_{2}\right)^{2}-m_{3}^{2}\right)}=B 0+m_{1}^{2} C 0 \\
& -i \int \frac{d^{4} l}{(2 \pi)^{4}} \frac{l^{\rho} l^{\sigma}}{\left.\left(l^{2}-m_{1}^{2}\right)\left(\left(l+v_{1}\right)^{2}-m_{2}^{2}\right)\left(l+v_{1}+v_{2}\right)^{2}-m_{3}^{2}\right)}=g^{\rho \sigma} C 24+C 21 v_{1}^{\rho} v_{1}^{\sigma} \\
& +C 22 v_{2}^{\rho} v_{2}^{\sigma}+C 23\left(v_{1}^{\rho} v_{2}^{\sigma}+v_{2}^{\rho} v_{1}^{\sigma}\right)(\mathrm{C} .1
\end{aligned}
$$


The arguments of the function $B 0$ are $\left(v_{2}^{2}, m_{2}^{2}, m_{3}^{2}\right)$.

For the form-factor of the scalar particles, exchanged in the $t$-channel, one substitutes, $0, m_{\chi_{a}}^{2}$, (or $\left.m_{\chi_{b}}^{2}\right), t$, for $v_{1}^{2}, v_{2}^{2},\left(v_{1}+v_{2}\right)^{2}$ respectively, whereas for the vector-boson form factors one substitutes $m_{\chi_{a}}^{2}, m_{\chi_{b}}^{2}, s$ for $v_{1}^{2}, v_{2}^{2},\left(v_{1}+v_{2}\right)^{2}$ respectively.

The contributions to the form-factors from each prototype vertex diagram are given below. Diagrams involving internal gauge-bosons are calculated in Feynman gauge.

\section{Scalar form-factor diagrams}

\section{VERTEX PROTOTYPE 1}

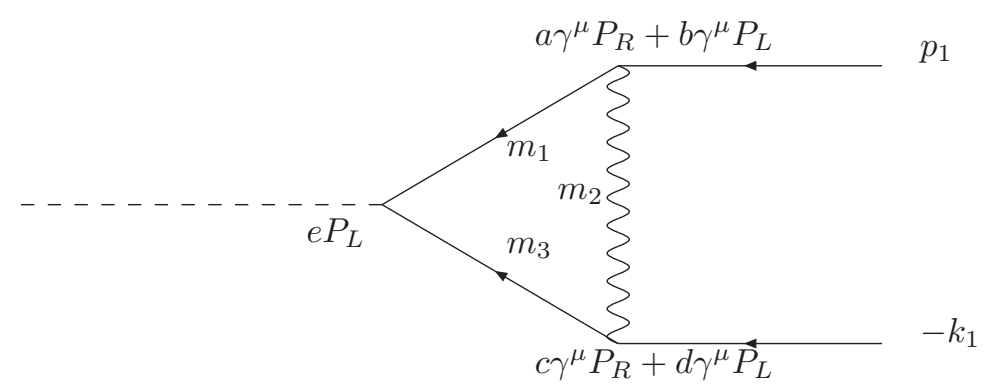

Figure 11: Vertex prototype graph 1.

$16 \pi^{2} \Delta F_{\tilde{\nu}}^{-}=b d e\left[2-4 B 0+2\left(m_{\chi_{a}}^{2}-t\right)(C 11+C 12)-4 m_{\chi_{a}}^{2} C 12\right]+2$ bce $m_{3} m_{\chi_{a}} C 12$

\section{VERTEX PROTOTYPE 2}

$16 \pi^{2} \Delta F_{\tilde{\nu}}^{-}=\operatorname{ade}\left[B 0+m_{1}^{2} C 0-t C 12\right]+$ ace $_{2} m_{\chi_{a}}[C 0-C 12]$

\section{VERTEX PROTOTYPE 3}

$16 \pi^{2} \Delta F_{\tilde{\nu}}^{-}=\operatorname{ade}\left[B 0+m_{1}^{2} C 0+2 m_{\chi_{a}}^{2} C 12+\left(t-m_{\chi_{a}}^{2}\right)(C 12+2 C 11-2 C 0)\right]$ 


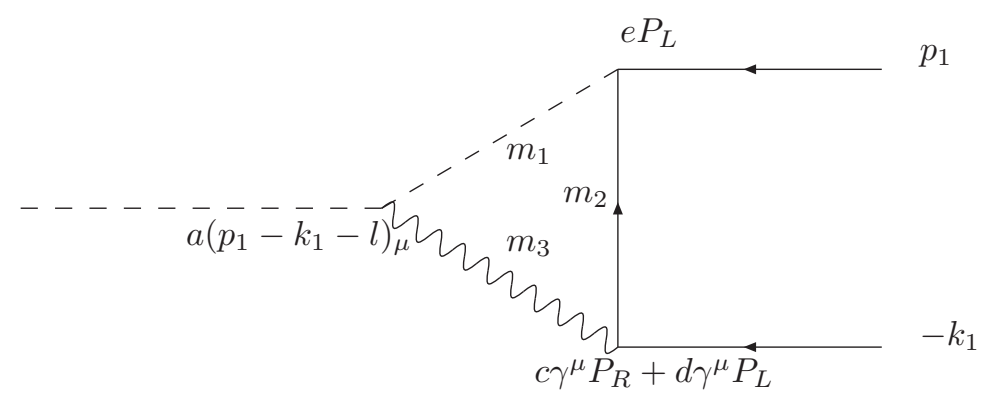

Figure 12: Vertex prototype graph 2.

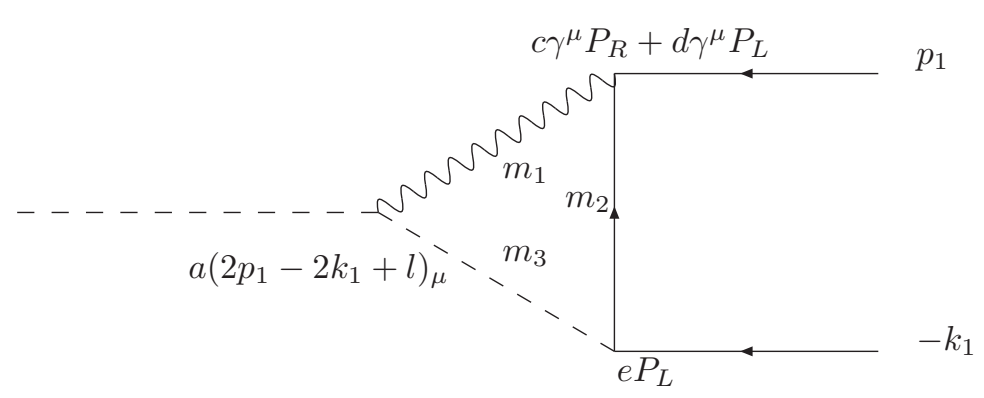

Figure 13: Vertex prototype graph 3.

\section{VERTEX PROTOTYPE 4}

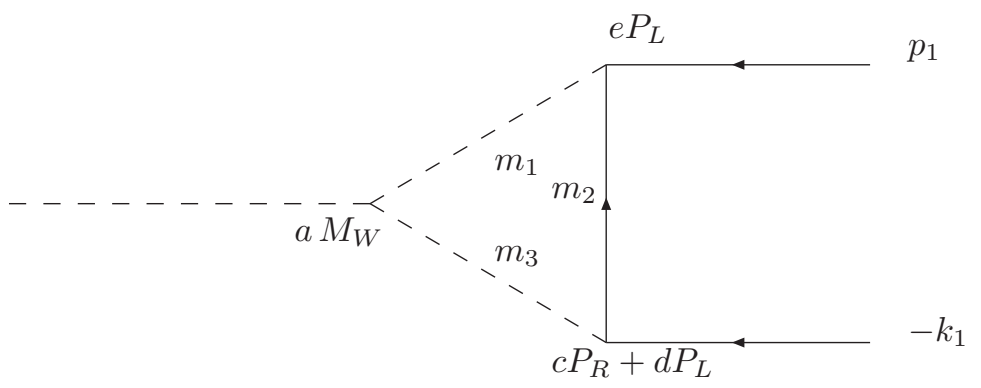

Figure 14: Vertex prototype graph 4.

$$
16 \pi^{2} \Delta F_{\tilde{\nu}}^{-}=2 \text { ade } M_{W} m_{2} C 0-2 \text { ace } M_{W} m_{\chi_{a}} C 12
$$

For all the above prototype graphs, we obtain similar expressions for $\Delta\left(F_{\tilde{\nu}}^{-}\right)^{\dagger}$ with $m_{\chi_{a}} \rightarrow m_{\chi_{b}}$. 


\section{Vector form-factor diagrams}

\section{VERTEX PROTOTYPE 5}

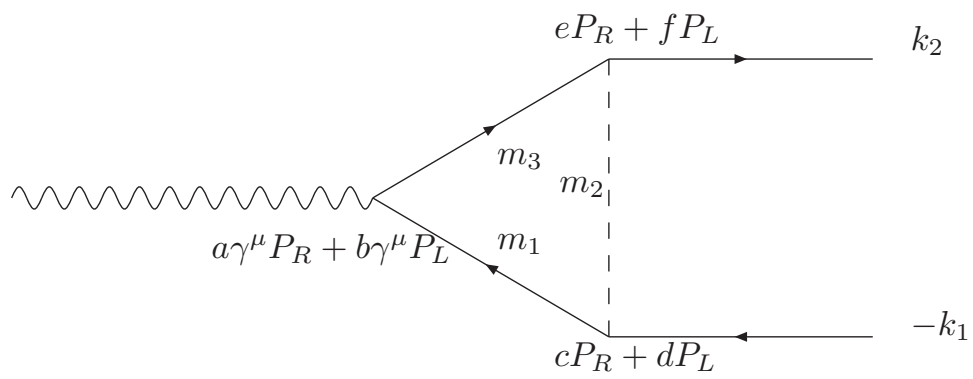

Figure 15: Vertex prototype graph 5.

$$
\begin{aligned}
16 \pi^{2} \Delta F_{0}^{+} & =\text {ace } m_{\chi_{b}} m_{3} C 12+b d f m_{\chi_{a}} m_{3} C 11 \\
& + \text { acf }\left[2 C 24-\frac{1}{2}+m_{\chi_{a}}^{2}(C 11-C 12+C 21-C 23)\right. \\
& \left.\quad+m_{\chi_{b}}^{2}(C 22-C 23)+s(C 12+C 23)\right] \\
& + \text { bde } m_{\chi_{a}} m_{\chi_{b}}[C 11-C 12]-\text { bcf } m_{1} m_{3} C 0
\end{aligned}
$$

$$
\begin{aligned}
16 \pi^{2} \Delta F_{k}^{+} & =\text {acf } m_{\chi_{b}}[C 22-C 23]+\text { bdem } m_{\chi_{a}}[C 11-C 12+C 21-C 23] \\
& + \text { ace } m_{3} C 12-\text { bce } m_{1}[C 0+C 11] \\
16 \pi^{2} \Delta F_{k}^{-} & =b d e m_{\chi_{b}}[C 22-C 23]+\text { acf } m_{\chi_{a}}[C 11-C 12+C 21-C 23] \\
& + \text { bdf } m_{3} C 12-\text { adf } m_{1}[C 0+C 11]
\end{aligned}
$$




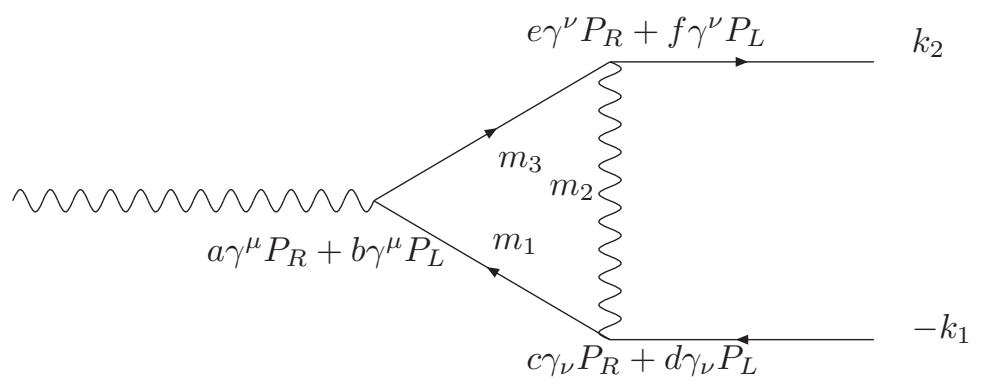

Figure 16: Vertex prototype graph 6.

\section{VERTEX PROTOTYPE 6}

$$
\begin{aligned}
& 16 \pi^{2} \Delta F_{0}^{+}=2 \text { ace }\left[2 C 24-1+m_{\chi_{a}}^{2}(C 21-C 23)\right. \\
& \left.+m_{\chi_{b}}^{2}(C 12-C 11+C 22-C 23)+s(C 11+C 23)\right] \\
& \text { - } 2 \text { bdf } m_{\chi_{a}} m_{\chi_{b}}[C 11-C 12]-2 \text { bce } m_{1} m_{3} C 0 \\
& 16 \pi^{2} \Delta F_{0}^{-}=2 b d f\left[2 C 24-1+m_{\chi_{a}}^{2}(C 21-C 23)\right. \\
& \left.+m_{\chi_{b}}^{2}(C 12-C 11+C 22-C 23)+s(C 11+C 23)\right] \\
& -2 \text { ace } m_{\chi_{a}} m_{\chi_{b}}[C 11-C 12]-2 \text { adf } m_{1} m_{3} C 0 \\
& 16 \pi^{2} \Delta F_{k}^{+}=2 b d f m_{\chi_{a}}[C 21-C 23]+2 \text { ace }_{\chi_{b}}[C 12-C 11+C 22-C 23] \\
& +2 b c f m_{1}[C 11-C 12]+2 a c f m_{3}[C 11-C 12] \\
& 16 \pi^{2} \Delta F_{k}^{-}=2 \text { acem }_{\chi_{a}}[C 21-C 23]+2 b d f m_{\chi_{b}}[C 12-C 11+C 22-C 23] \\
& +2 \text { ade }_{1}[C 11-C 12]+2 \text { bde } m_{3}[C 11-C 12]
\end{aligned}
$$

\section{VERTEX PROTOTYPE 7}

$a_{\nu \rho}^{\mu}$ is the usual triple gauge-boson vertex, multiplied by a gauge coupling, $a$.

$$
\begin{gathered}
16 \pi^{2} \Delta F_{0}^{+}=\text {ace }[ \\
{\left[4 C 24+2 B 0-1+2 m_{1}^{2} C 0+m_{\chi_{a}}^{2}(2 C 0+4 C 11-3 C 12)\right.} \\
\left.+m_{\chi_{b}}^{2}(C 0+C 11-2 C 12)+s(3 C 12-C 11-C 0)\right] \\
+3 a d f m_{\chi_{a}} m_{\chi_{b}}[C 0+C 11-C 12]-3 a c f m_{2} m_{\chi_{b}} C 0-3 a d e m_{2} m_{\chi_{a}} C 0(\mathrm{C} .14)
\end{gathered}
$$




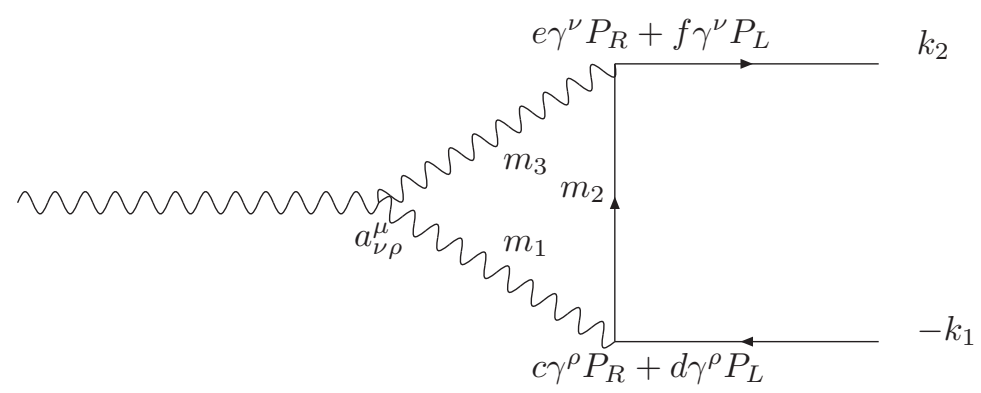

Figure 17: Vertex prototype graph 7.

$$
\begin{aligned}
& 16 \pi^{2} \Delta F_{0}^{-}=\operatorname{adf}\left[4 C 24+2 B 0-1+2 m_{1}^{2} C 0+m_{\chi_{a}}^{2}(2 C 0+4 C 11-3 C 12)\right. \\
& \left.+m_{\chi_{b}}^{2}(C 0+C 11-2 C 12)+s(3 C 12-C 11-C 0)\right] \\
& +3 \text { ace }_{\chi_{a}} m_{\chi_{b}}[C 0+C 11-C 12]-3 \text { ade } m_{2} m_{\chi_{b}} C 0-3 \text { acf } m_{2} m_{\chi_{a}} C 0 \\
& 16 \pi^{2} \Delta F_{k}^{+}=\text {acem }_{\chi_{b}}[2 C 0+2 C 11-C 12-2 C 22+2 C 23] \\
& +\operatorname{adf} m_{\chi_{a}}[C 0-C 11-2 C 21+2 C 23]-3 a c f m_{2}[C 0-C 11+C 12](\text { C.16) } \\
& 16 \pi^{2} \Delta F_{k}^{-}=a d f m_{\chi_{b}}[2 C 0+2 C 11-C 12-2 C 22+2 C 23] \\
& + \text { acem }_{\chi_{a}}[C 0-C 11-2 C 21+2 C 23]-3 \text { adem }_{2}[C 0-C 11+C 12](\text { C.17) }
\end{aligned}
$$

\section{VERTEX PROTOTYPE 8}

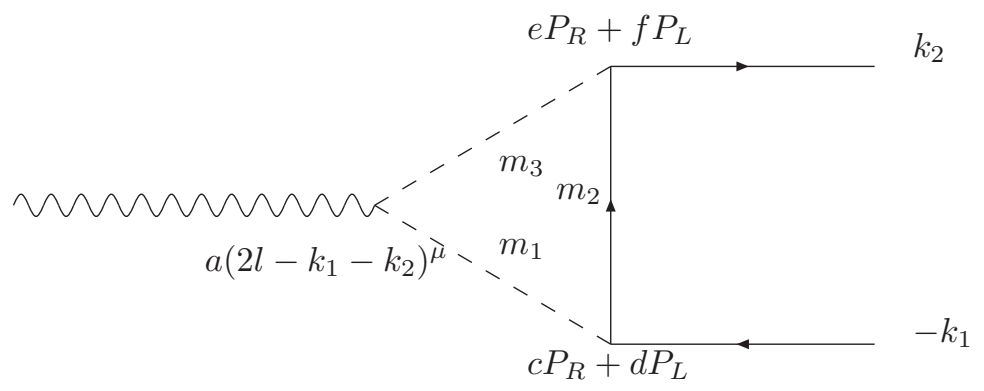

Figure 18: Vertex prototype graph 8.

$$
16 \pi^{2} \Delta F_{0}^{+}=-2 a c f C 24
$$




$$
\begin{aligned}
16 \pi^{2} \Delta F_{0}^{-}=-2 \operatorname{ade} C 24 \\
16 \pi^{2} \Delta F_{k}^{+}=\operatorname{acf} m_{\chi_{b}}[C 22-C 23]+\operatorname{adem}_{\chi_{a}}[C 11-C 12+C 21-C 23] \\
+\operatorname{acem} m_{2}[C 11-C 12] \\
16 \pi^{2} \Delta F_{k}^{-}=\operatorname{adem}_{\chi_{b}}[C 22-C 23]+\operatorname{acf} m_{\chi_{a}}[C 11-C 12+C 21-C 23] \\
+ \text { adf } m_{2}[C 11-C 12]
\end{aligned}
$$

\section{Appendix D}

\section{Prototype box graphs}

In this Appendix we give the expressions for the prototype box diagrams, with couplings indicated in the diagrams, in terms of the Veltman-Passarino functions, $D 0, D 1(i), i=1 \cdots 3, D 20$ and $D 2(i, j), i, j=1 \cdots 3$. These are defined as

$$
\begin{aligned}
& -i \int \frac{d^{4} l}{\pi^{2}} \frac{1}{\left.\left(l^{2}-m_{1}^{2}\right)\left(\left(l+v_{1}\right)^{2}-m_{2}^{2}\right)\left(l+v_{1}+v_{2}\right)^{2}-m_{3}^{2}\right)\left(\left(l-v_{4}\right)^{2}-m_{4}^{2}\right)}=D 0 \\
& -i \int \frac{d^{4} l}{\pi^{2}} \frac{l^{\rho}}{\left.\left(l^{2}-m_{1}^{2}\right)\left(\left(l+v_{1}\right)^{2}-m_{2}^{2}\right)\left(l+v_{1}+v_{2}\right)^{2}-m_{3}^{2}\right)\left(\left(l-v_{4}\right)^{2}-m_{4}^{2}\right)}=\sum_{i=1}^{3} D 1(i) v_{i}^{\rho}, \\
& -i \int \frac{d^{4} l}{\pi^{2}} \frac{l^{2}}{\left.\left(l^{2}-m_{1}^{2}\right)\left(\left(l+v_{1}\right)^{2}-m_{2}^{2}\right)\left(l+v_{1}+v_{2}\right)^{2}-m_{3}^{2}\right)\left(\left(l-v_{4}\right)^{2}-m_{4}^{2}\right)}=C 0(1)+m_{1}^{2} D 0 \\
& -i \int \frac{d^{4} l}{\pi^{2}} \frac{l^{\rho} l^{\sigma}}{\left.\left(l^{2}-m_{1}^{2}\right)\left(\left(l+v_{1}\right)^{2}-m_{2}^{2}\right)\left(l+v_{1}+v_{2}\right)^{2}-m_{3}^{2}\right)\left(\left(l-v_{4}\right)^{2}-m_{4}^{2}\right)}=g^{\rho \sigma} D 20 \\
& +\sum_{i, j=1}^{3} D 2(i, j) v_{i}^{\rho} v_{j}^{\sigma} \quad \text { (D.1) }
\end{aligned}
$$

For the uncrossed box prototypes 1, 2, 3a, 3b, 4a the vectors $v_{i}$ are

$$
\begin{gathered}
v_{1}^{\mu}=p_{1}^{\mu} \\
v_{2}^{\mu}=p_{2}^{\mu} \\
v_{3}^{\mu}=-k_{2}^{\mu} \\
v_{4}^{\mu}=-k_{1}^{\mu}
\end{gathered}
$$

The arguments of the D-functions are

$$
\operatorname{Di}(\alpha)=\operatorname{Di}(\alpha)\left(s, t, 0,0, m_{\chi_{b}}^{2}, m_{\chi_{a}}^{2}, m_{1}, m_{2}, m_{3}, m_{4}\right)
$$


and $C 0(1)$ means

$$
C 0\left(0, m_{\chi_{b}}^{2}, t, m_{2}^{2}, m_{3}^{2}, m_{4}^{2}\right),
$$

whereas for the crossed box prototypes $4 \mathrm{c}, 5 \mathrm{a}$ and $5 \mathrm{~b}$

$$
\begin{gathered}
v_{1}^{\mu}=p_{1}^{\mu} \\
v_{2}^{\mu}=-k_{2}^{\mu} \\
v_{3}^{\mu}=p_{2}^{\mu} \\
v_{4}^{\mu}=-k_{1}^{\mu} .
\end{gathered}
$$

The arguments of the D-functions are

$$
D i(\alpha)=D i(\alpha)\left(u, t, 0, m_{\chi_{b}}^{2}, 0, m_{\chi_{a}}^{2}, m_{1}, m_{2}, m_{3}, m_{4}\right)
$$

and $C 0(1)$ means

$$
C 0\left(m_{\chi_{b}}^{2}, 0, t, m_{2}^{2}, m_{3}^{2}, m_{4}^{2}\right)
$$

The contributions, $\Delta \mathcal{Q}_{\alpha, i, j}$ to the coefficient function $\mathcal{Q}_{\alpha, i, j}$ from each prototype box diagram are given below. Diagrams involving internal gauge-bosons are calculated in Feynman gauge.

\section{BOX PROTOTYPE 1}

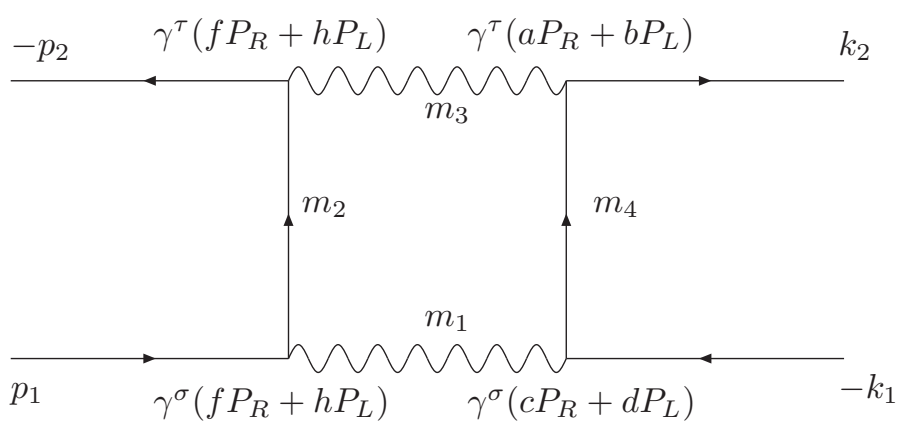

Figure 19: Box prototype graph 1.

$$
\begin{aligned}
\frac{16 \pi^{2}}{s} \Delta \mathcal{Q}_{R, 1} & =m_{\chi_{a}} b d f^{2}[D 0+D 1(1)+D 1(2)+D 1(3)+D 2(3,1)+D 2(3,2)] \\
& -m_{4} b c f^{2}[D 0+D 1(1)+D 1(2)]
\end{aligned}
$$




$$
\begin{aligned}
\frac{16 \pi^{2}}{s} \Delta \mathcal{Q}_{R, 2}= & -m_{\chi_{b}} b d f^{2}[D 0+D 1(1)+D 1(2)-D 1(3) \\
& +D 2(3,1)+D 2(3,2)-2 D 2(3,3)] \\
& +m_{4} a d f^{2}[D 0+D 1(1)+D 1(2)-2 D 1(3)]
\end{aligned}
$$

$$
\begin{aligned}
& \frac{16 \pi^{2}}{s} \Delta \mathcal{Q}_{R, 3,1}=m_{\chi_{a}} m_{4} a d f^{2}[D 0+D 1(1)+D 1(2)] \\
&-2 m_{\chi_{a}}^{2} a c f^{2}[D 0+D 1(1)+D 1(2)+D 1(3)] \\
&-m_{\chi_{b}} m_{4} b c f^{2}[D 0+D 1(1)+D 1(2)-2 D 1(3)] \\
&-4 m_{1}^{2} a c f^{2} D 0 \\
&+a c f^{2}[2 t(D 0+D 1(1))-4 C 0(1)-2(s-u)(D 1(2)-D 1(3))] \\
& \frac{16 \pi^{2}}{s} \Delta \mathcal{Q}_{R, 3,2}=0
\end{aligned}
$$

$$
\begin{aligned}
\frac{16 \pi^{2}}{s} \Delta \mathcal{Q}_{R, 4,1} & =-m_{\chi_{a}} m_{4} b c f^{2}[D 0+D 1(1)+D 1(2)] \\
& +m_{\chi_{b}} m_{4} a d f^{2}[(D 0+D 1(1)+D 1(2)-2 D 1(3)] \\
& -4 b d f^{2} D 20
\end{aligned}
$$

$$
\frac{16 \pi^{2}}{s} \Delta \mathcal{Q}_{R, 4,2}=-b d f^{2}[D 0+D 1(1)-D 1(2)+D 1(3)+D 2(3,1)-D 2(3,2)]
$$

$$
\frac{16 \pi^{2}}{s} \Delta \mathcal{Q}_{R, 5,1}=-\frac{1}{2} m_{4}(a d-b c) f^{2}[D 0+D 1(1)-D 1(2)]
$$

$$
\frac{16 \pi^{2}}{s} \Delta \mathcal{Q}_{R, 5,2}=-\frac{1}{4} m_{4}(a d+b c) f^{2}[D 0+D 1(1)-D 1(2)]
$$

$$
\begin{aligned}
\frac{16 \pi^{2}}{s} \Delta \mathcal{Q}_{L, 1}= & -m_{\chi_{b}} a c h^{2}[D 0+D 1(1)+D 1(2)-D 1(3) \\
& +D 2(3,1)+D 2(3,2)-2 D 2(3,3)] \\
& +m_{4} b c h^{2}[D 0+D 1(1)+D 1(2)-2 D 1(3)]
\end{aligned}
$$

$$
\begin{aligned}
\frac{16 \pi^{2}}{s} \Delta \mathcal{Q}_{L, 2} & =m_{\chi_{a}} a c h^{2}[D 0+D 1(1)+D 1(2)+D 1(3)+D 2(3,1)+D 2(3,2)] \\
& -m_{4} a d h^{2}[D 0+D 1(1)+D 1(2)]
\end{aligned}
$$




$$
\begin{aligned}
\frac{16 \pi^{2}}{s} \Delta \mathcal{Q}_{L, 3,1} & =-m_{\chi_{a}} m_{4} a d h^{2}[D 0+D 1(1)+D 1(2)] \\
& +m_{\chi_{b}} m_{4} b c h^{2}[(D 0+D 1(1)+D 1(2)-2 D 1(3)] \\
& -4 a c h^{2} D 20
\end{aligned}
$$

$\frac{16 \pi^{2}}{s} \Delta \mathcal{Q}_{L, 3,2}=-a c h^{2}[D 0+D 1(1)-D 1(2)+D 1(3)+D 2(3,1)-D 2(3,2)]$

$$
\begin{aligned}
\frac{16 \pi^{2}}{s} \Delta \mathcal{Q}_{L, 4,1} & =m_{\chi_{a}} m_{4} b c h^{2}[D 0+D 1(1)+D 1(2)] \\
& -2 m_{\chi_{a}}^{2} b d h^{2}[D 0+D 1(1)+D 1(2)+D 1(3)] \\
& -m_{\chi_{b}} m_{4} a d h^{2}[D 0+D 1(1)+D 1(2)-2 D 1(3)] \\
& -4 m_{1}^{2} b d h^{2} D 0 \\
+b d h^{2}(2 t & (D 0+D 1(1))-4 C 0(1)-2(s-u)(D 1(2)-D 1(3))]
\end{aligned}
$$

$$
\frac{16 \pi^{2}}{s} \Delta \mathcal{Q}_{L, 4,2}=0
$$

$$
\begin{aligned}
& \frac{16 \pi^{2}}{s} \Delta \mathcal{Q}_{L, 5,1}=\frac{1}{2} m_{4}(a d-b c) h^{2}[D 0+D 1(1)-D 1(2)] \\
& \frac{16 \pi^{2}}{s} \Delta \mathcal{Q}_{L, 5,2}=\frac{1}{4} m_{4}(a d+b c) h^{2}[D 0+D 1(1)-D 1(2)]
\end{aligned}
$$

\section{BOX PROTOTYPE 2}

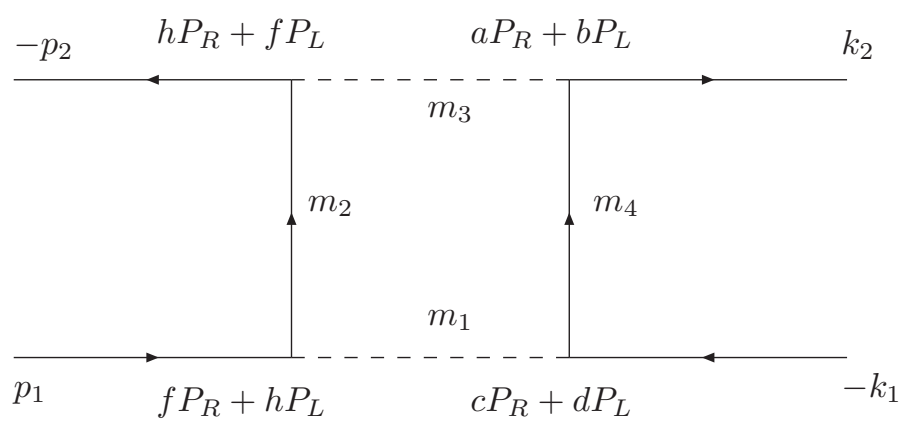

Figure 20: Box prototype graph 2. 


$$
\begin{aligned}
\frac{16 \pi^{2}}{s} \Delta \mathcal{Q}_{R, 1} & =\frac{1}{4} m_{\chi_{a}} a d f^{2}[2 D 1(3)+D 2(3,1)+D 2(3,2)] \\
& +\frac{1}{4} m_{\chi_{b}} b c f^{2}[2 D 2(3,3)-D 2(3,1)-D 2(3,2)] \\
& +\frac{1}{2} m_{4} a c f^{2} D 1(3)
\end{aligned}
$$

$$
\begin{aligned}
\frac{16 \pi^{2}}{s} \Delta \mathcal{Q}_{R, 2}= & \frac{1}{4} m_{\chi_{a}} b c f^{2}[2 D 1(3)+D 2(3,1)+D 2(3,2)] \\
+ & \frac{1}{4} m_{\chi_{b}} a d f^{2}[2 D 2(3,3)-D 2(3,1)-D 2(3,2)] \\
+ & \frac{1}{2} m_{4} b d f^{2} D 1(3) \\
& \frac{16 \pi^{2}}{s} \Delta \mathcal{Q}_{R, 3,1}=-b c f^{2} D 20
\end{aligned}
$$

$$
\begin{gathered}
\frac{16 \pi^{2}}{s} \Delta \mathcal{Q}_{R, 3,2}=\frac{1}{4} b c f^{2}[D 2(3,2)-D 2(3,1)] \\
\frac{16 \pi^{2}}{s} \Delta \mathcal{Q}_{R, 4,1}=-a d f^{2} D 20
\end{gathered}
$$$$
\frac{16 \pi^{2}}{s} \Delta \mathcal{Q}_{R, 4,2}=\frac{1}{4} a d f^{2}[D 2(3,2)-D 2(3,1)]
$$$$
\frac{16 \pi^{2}}{s} \Delta \mathcal{Q}_{R, 5,1}=0
$$$$
\frac{16 \pi^{2}}{s} \Delta \mathcal{Q}_{R, 5,2}=0
$$

$$
\begin{aligned}
\frac{16 \pi^{2}}{s} \Delta \mathcal{Q}_{L, 1} & =\frac{1}{4} m_{\chi_{a}} a d h^{2}[2 D 1(3)+D 2(3,1)+D 2(3,2)] \\
& +\frac{1}{4} m_{\chi_{b}} b c h^{2}[2 D 2(3,3)-D 2(3,1)-D 2(3,2)] \\
& +\frac{1}{2} m_{4} a c h^{2} D 1(3)
\end{aligned}
$$

$$
\begin{aligned}
\frac{16 \pi^{2}}{s} \Delta \mathcal{Q}_{L, 2} & =\frac{1}{4} m_{\chi_{a}} b c h^{2}[2 D 1(3)+D 2(3,1)+D 2(3,2)] \\
& +\frac{1}{4} m_{\chi_{b}} a d h^{2}[2 D 2(3,3)-D 2(3,1)-D 2(3,2)] \\
& +\frac{1}{2} m_{4} b d h^{2} D 1(3)
\end{aligned}
$$




$$
\begin{gathered}
\frac{16 \pi^{2}}{s} \Delta \mathcal{Q}_{L, 3,1}=-b c h^{2} D 20 \\
\frac{16 \pi^{2}}{s} \Delta \mathcal{Q}_{L, 3,2}=\frac{1}{4} b c h^{2}[D 2(3,2)-D 2(3,1)] \\
\frac{16 \pi^{2}}{s} \Delta \mathcal{Q}_{L, 4,1}=-a d h^{2} D 20 \\
\frac{16 \pi^{2}}{s} \Delta \mathcal{Q}_{L, 4,2}=\frac{1}{4} a d h^{2}[D 2(3,2)-D 2(3,1)] \\
\frac{16 \pi^{2}}{s} \Delta \mathcal{Q}_{L, 5,1}=0 \\
\frac{16 \pi^{2}}{s} \Delta \mathcal{Q}_{L, 5,2}=0
\end{gathered}
$$

\section{BOX PROTOTYPE 3a}

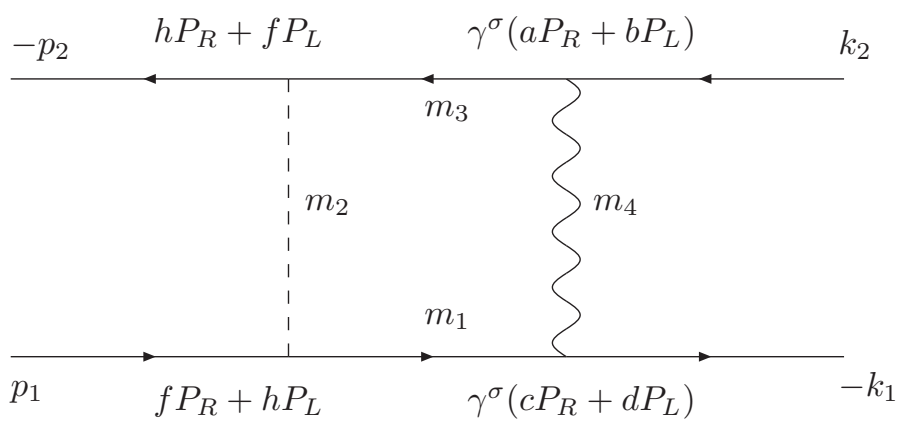

Figure 21: Box prototype graph 3a.

$$
\begin{aligned}
\frac{16 \pi^{2}}{s} \Delta \mathcal{Q}_{R, 1} & =-\frac{1}{2} m_{\chi_{a}} b d f^{2}[2 D 1(3)+D 2(3,1)+D 2(3,2)] \\
& +m_{3} a d f^{2} D 1(3) \\
\frac{16 \pi^{2}}{s} \Delta \mathcal{Q}_{R, 2} & =\frac{1}{2} m_{\chi_{b}} b d f^{2}[D 2(3,1)+D 2(3,2)-2 D 2(3,3)] \\
& +m_{1} b c f^{2} D 1(3)
\end{aligned}
$$




$$
\begin{aligned}
& \frac{16 \pi^{2}}{s} \Delta \mathcal{Q}_{R, 3,1}=-m_{\chi_{a}} m_{\chi_{b}} b d f^{2} D 1(3)+m_{1} m_{3} a c f^{2} D 0 \\
& \frac{16 \pi^{2}}{s} \Delta \mathcal{Q}_{R, 3,2}=0 \\
& \frac{16 \pi^{2}}{s} \Delta \mathcal{Q}_{R, 4,1}=\frac{1}{2} b d f^{2}\left(m_{\chi_{b}}^{2}-m_{\chi_{a}}^{2}\right) D 1(3)-m_{1}^{2} b d f^{2} D 0 \\
& +\frac{1}{2} b d f^{2}[4 D 20-2 C 0(1)-(s-t+u) D 1(1) \\
& -(s+t-u) D 1(2)+(s-t-u) D 1(3)] \\
& \frac{16 \pi^{2}}{s} \Delta \mathcal{Q}_{R, 4,2}=\frac{1}{2} b d f^{2}[D 1(1)-D 1(2)+D 2(3,1)-D 2(3,2)] \\
& \frac{16 \pi^{2}}{s} \Delta \mathcal{Q}_{R, 5,1}=\frac{1}{4} b d f^{2}\left(m_{\chi_{b}}-m_{\chi_{a}}\right)[D 1(1)-D 1(2)] \\
& \frac{16 \pi^{2}}{s} \Delta \mathcal{Q}_{R, 5,2}=\frac{1}{8} b d f^{2}\left(m_{\chi_{b}}+m_{\chi_{a}}\right)[D 1(1)-D 1(2)] \\
& \frac{16 \pi^{2}}{s} \Delta \mathcal{Q}_{L, 1}=\frac{1}{2} m_{\chi_{b}} a c h^{2}[D 2(3,1)+D 2(3,2)-2 D 2(3,3)] \\
& +m_{1} a d h^{2} D 1(3) \\
& \frac{16 \pi^{2}}{s} \Delta \mathcal{Q}_{L, 2}=-\frac{1}{2} m_{\chi_{a}} a c h^{2}[2 D 1(3)+D 2(3,1)+D 2(3,2)] \\
& +m_{3} b c h^{2} D 1(3) \\
& \frac{16 \pi^{2}}{s} \Delta \mathcal{Q}_{L, 3,1}=\frac{1}{2} a c h^{2}\left(m_{\chi_{b}}^{2}-m_{\chi_{a}}^{2}\right) D 1(3)-m_{1}^{2} a c h^{2} D 0 \\
& +\frac{1}{2} a c h^{2}[4 D 20-2 C 0(1)-(s-t+u) D 1(1) \\
& -(s+t-u) D 1(2)+(s-t-u) D 1(3)] \\
& \frac{16 \pi^{2}}{s} \Delta \mathcal{Q}_{L, 3,2}=\frac{1}{2} a c h^{2}[D 1(1)-D 1(2)+D 2(3,1)-D 2(3,2)]
\end{aligned}
$$




$$
\begin{gathered}
\frac{16 \pi^{2}}{s} \Delta \mathcal{Q}_{L, 4,1}=-m_{\chi_{a}} m_{\chi_{b}} a c h^{2} D 1(3)+m_{1} m_{3} b d h^{2} D 0 \\
\frac{16 \pi^{2}}{s} \Delta \mathcal{Q}_{L, 4,2}=0 \\
\frac{16 \pi^{2}}{s} \Delta \mathcal{Q}_{L, 5,1}=\frac{1}{4} a c h^{2}\left(m_{\chi_{b}}-m_{\chi_{a}}\right)[D 1(1)-D 1(2)] \\
\frac{16 \pi^{2}}{s} \Delta \mathcal{Q}_{L, 5,2}=-\frac{1}{8} a c h^{2}\left(m_{\chi_{b}}+m_{\chi_{a}}\right)[D 1(1)-D 1(2)]
\end{gathered}
$$

\section{BOX PROTOTYPE 3b}

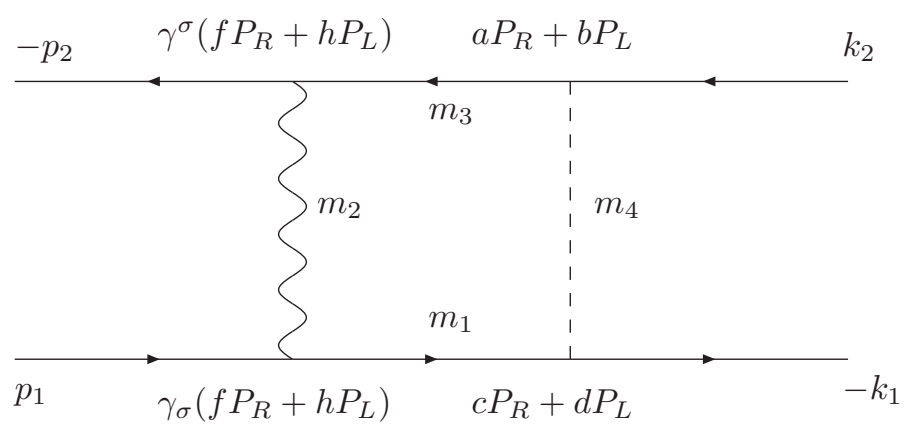

Figure 22: Box prototype graph 3b.

$$
\begin{gathered}
\frac{16 \pi^{2}}{s} \Delta \mathcal{Q}_{R, 1}=-\frac{1}{2} m_{\chi_{a}} b c f^{2}[D 2(3,1)+D 2(3,2)] \\
-\frac{1}{2} m_{3} a c f^{2}[D 1(1)+D 1(2)] \\
\frac{16 \pi^{2}}{s} \Delta \mathcal{Q}_{R, 2}=\frac{1}{2} m_{\chi_{b}} b c f^{2}[2 D 1(3)+D 2(3,1)+D 2(3,2)-2 D 2(3,3)] \\
+\frac{1}{2} m_{1} b d f^{2}[2 D 0+D 1(1)+D 1(2)-2 D 1(3)] \\
\frac{16 \pi^{2}}{s} \Delta \mathcal{Q}_{R, 3,1}=m_{\chi_{a}} m_{\chi_{b}} b c f^{2} D 1(3)+m_{1} m_{3} a d f^{2} D 0 \\
+\frac{1}{2} m_{\chi_{a}} m_{1} b d f^{2}[2 D 0+D 1(1)+D 1(2)] \\
+\frac{1}{2} m_{\chi_{b}} m_{3} a c f^{2}[2 D 1(3)-D 1(1)-D 1(2)]
\end{gathered}
$$




$$
\begin{aligned}
& \frac{16 \pi^{2}}{s} \Delta \mathcal{Q}_{R, 3,2}=0 \\
& \frac{16 \pi^{2}}{s} \Delta \mathcal{Q}_{R, 4,1}=\frac{1}{2} m_{\chi_{b}} m_{1} b d f^{2}[2 D 0+D 1(1)+D 1(2)-2 D 1(3)] \\
& -\frac{1}{2} m_{\chi_{a}} m_{3} a c f^{2}[D 1(1)+D 1(2)]-m_{1}^{2} b c f^{2} D 0 \\
& +\frac{1}{2} b c f^{2}[4 D 20-2 C 0(1)-(s+t-u) D 1(1) \\
& \left.-(s-t+u) D 1(2)+2 m_{\chi_{b}}^{2} D 1(3)\right] \\
& \frac{16 \pi^{2}}{s} \Delta \mathcal{Q}_{R, 4,2}=-\frac{1}{2} b c f^{2}[D 1(1)-D 1(2)-D 2(3,1)+D 2(3,2)] \\
& \frac{16 \pi^{2}}{s} \Delta \mathcal{Q}_{R, 5,1}=\frac{1}{4} f^{2}\left(\left(m_{\chi_{a}}-m_{\chi_{b}}\right) b c-m_{1} b d+m_{3} a c\right)[D 1(1)-D 1(2)] \\
& \frac{16 \pi^{2}}{s} \Delta \mathcal{Q}_{R, 5,2}=-\frac{1}{8} f^{2}\left(\left(m_{\chi_{a}}+m_{\chi_{b}}\right) b c+m_{1} b d+m_{3} a c\right)[D 1(1)-D 1(2)] \\
& \frac{16 \pi^{2}}{s} \Delta \mathcal{Q}_{L, 1}=\frac{1}{2} m_{\chi_{b}} a d h^{2}[2 D 1(3)+D 2(3,1)+D 2(3,2)-2 D 2(3,3)] \\
& +\frac{1}{2} m_{1} a c h^{2}[2 D 0+D 1(1)+D 1(2)-2 D 1(3)] \\
& \frac{16 \pi^{2}}{s} \Delta \mathcal{Q}_{L, 2}=-\frac{1}{2} m_{\chi_{a}} a d h^{2}[D 2(3,1)+D 2(3,2)] \\
& -\frac{1}{2} m_{3} b d h^{2}[D 1(1)+D 1(2)] \\
& \frac{16 \pi^{2}}{s} \Delta \mathcal{Q}_{L, 3,1}=\frac{1}{2} m_{\chi_{b}} m_{1} a c h^{2}[2 D 0+D 1(1)+D 1(2)-2 D 1(3)] \\
& -\frac{1}{2} m_{\chi_{a}} m_{3} b d h^{2}[D 1(1)+D 1(2)]-m_{1}^{2} a d h^{2} D 0 \\
& +\frac{1}{2} a d h^{2}[4 D 20-2 C 0(1)-(s+t-u) D 1(1) \\
& \left.-(s-t+u) D 1(2)+2 m_{\chi_{b}}^{2} D 1(3)\right]
\end{aligned}
$$




$$
\begin{aligned}
& \frac{16 \pi^{2}}{s} \Delta \mathcal{Q}_{L, 3,2}=-\frac{1}{2} a d h^{2}[D 1(1)-D 1(2)-D 2(3,1)+D 2(3,2)] \\
& \frac{16 \pi^{2}}{s} \Delta \mathcal{Q}_{L, 4,1}=m_{\chi_{a}} m_{\chi_{b}} a d h^{2} D 1(3)+m_{1} m_{3} b c h^{2} D 0 \\
& +\frac{1}{2} m_{\chi_{a}} m_{1} a c h^{2}[2 D 0+D 1(1)+D 1(2)] \\
& +\frac{1}{2} m_{\chi_{b}} m_{3} b d h^{2}[2 D 1(3)-D 1(1)-D 1(2)] \\
& \frac{16 \pi^{2}}{s} \Delta \mathcal{Q}_{L, 4,2}=0 \\
& \frac{16 \pi^{2}}{s} \Delta \mathcal{Q}_{L, 5,1}=\frac{1}{4} h^{2}\left(\left(m_{\chi_{a}}-m_{\chi_{b}}\right) a d-m_{1} a c+m_{3} b d\right)[D 1(1)-D 1(2)] \\
& \frac{16 \pi^{2}}{s} \Delta \mathcal{Q}_{L, 5,2}=\frac{1}{8} h^{2}\left(\left(m_{\chi_{a}}+m_{\chi_{b}}\right) a d+m_{1} a c+m_{3} b d\right)[D 1(1)-D 1(2)]
\end{aligned}
$$

\section{BOX PROTOTYPE 4a}

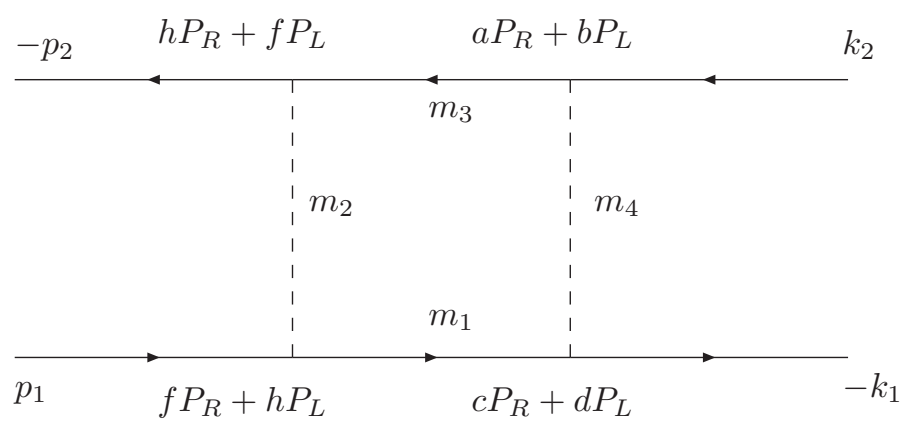

Figure 23: Box prototype graph 4a.

$$
\begin{aligned}
\frac{16 \pi^{2}}{s} \Delta \mathcal{Q}_{R, 1} & =\frac{1}{4} m_{\chi_{b}} a d f^{2}[2 D 1(3)+D 2(3,1)+D 2(3,2)-2 D 2(3,3)] \\
& +\frac{1}{4} m_{1} a c f^{2}[2 D 0+D 1(1)+D 1(2)-2 D 1(3)]
\end{aligned}
$$




$$
\begin{aligned}
& \frac{16 \pi^{2}}{s} \Delta \mathcal{Q}_{R, 2}=-\frac{1}{4} m_{\chi_{a}} a d f^{2}[D 2(3,1)+D 2(3,2)] \\
& -\frac{1}{4} m_{3} b d f^{2}[D 1(1)+D 1(2)] \\
& \frac{16 \pi^{2}}{s} \Delta \mathcal{Q}_{R, 3,1}=\frac{1}{4} m_{\chi_{b}} m_{1} a c f^{2}[2 D 0+D 1(1)+D 1(2)-2 D 1(3)] \\
& -\frac{1}{4} m_{\chi_{a}} m_{3} b d f^{2}[D 1(1)+D 1(2)]-\frac{1}{2} m_{1}^{2} a d f^{2} D 0 \\
& +\frac{1}{4} a d f^{2}[4 D 20-2 C 0(1)-(s+t-u) D 1(1) \\
& \left.-(s-t+u) D 1(2)+2 m_{\chi_{b}}^{2} D 1(3)\right] \\
& \frac{16 \pi^{2}}{s} \Delta \mathcal{Q}_{R, 3,2}=-\frac{1}{4} a d f^{2}[D 1(1)-D 1(2)-D 2(3,1)+D 2(3,2)] \\
& \frac{16 \pi^{2}}{s} \Delta \mathcal{Q}_{R, 4,1}=\frac{1}{2} m_{\chi_{a}} m_{\chi_{b}} a d f^{2} D 1(3)+\frac{1}{2} m_{1} m_{3} b c f^{2} D 0 \\
& +\frac{1}{4} m_{\chi_{a}} m_{1} a c f^{2}[2 D 0+D 1(1)+D 1(2)] \\
& +\frac{1}{4} m_{\chi_{b}} m_{3} b d f^{2}[2 D 1(3)-D 1(1)-D 1(2)] \\
& \frac{16 \pi^{2}}{s} \Delta \mathcal{Q}_{R, 4,2}=0 \\
& \frac{16 \pi^{2}}{s} \Delta \mathcal{Q}_{R, 5,1}=\frac{1}{8} f^{2}\left(\left(m_{\chi_{a}}-m_{\chi_{b}}\right) a d-m_{1} a c+m_{3} b d\right)[D 1(1)-D 1(2)] \\
& \frac{16 \pi^{2}}{s} \Delta \mathcal{Q}_{R, 5,2}=\frac{1}{16} f^{2}\left(\left(m_{\chi_{a}}+m_{\chi_{b}}\right) a d+m_{1} a c+m_{3} b d\right)[D 1(1)-D 1(2)] \\
& \frac{16 \pi^{2}}{s} \Delta \mathcal{Q}_{L, 1}=-\frac{1}{4} m_{\chi_{a}} b c h^{2}[D 2(3,1)+D 2(3,2)] \\
& -\frac{1}{4} m_{3} a c h^{2}[D 1(1)+D 1(2)]
\end{aligned}
$$




$$
\begin{gathered}
\frac{16 \pi^{2}}{s} \Delta \mathcal{Q}_{L, 2}=\frac{1}{4} m_{\chi_{b}} b c h^{2}[2 D 1(3)+D 2(3,1)+D 2(3,2)-2 D 2(3,3)] \\
+\frac{1}{4} m_{1} b d h^{2}[2 D 0+D 1(1)+D 1(2)-2 D 1(3)] \\
\frac{16 \pi^{2}}{s} \Delta \mathcal{Q}_{L, 3,1}=\frac{1}{2} m_{\chi_{a}} m_{\chi_{b}} b c h^{2} D 1(3)+\frac{1}{2} m_{1} m_{3} a d h^{2} D 0 \\
\quad+\frac{1}{4} m_{\chi_{a}} m_{1} b d h^{2}[2 D 0+D 1(1)+D 1(2)] \\
\quad+\frac{1}{4} m_{\chi_{b}} m_{3} a c h^{2}[2 D 1(3)-D 1(1)-D 1(2)] \\
\frac{16 \pi^{2}}{s} \Delta \mathcal{Q}_{L, 3,2}=0 \\
\frac{16 \pi^{2} \Delta \mathcal{Q}_{L, 4,1}=}{s} \frac{1}{4} m_{\chi_{b}} m_{1} b d h^{2}[2 D 0+D 1(1)+D 1(2)-2 D 1(3)] \\
-\frac{1}{4} m_{\chi_{a}} m_{3} a c h^{2}[D 1(1)+D 1(2)]-\frac{1}{2} m_{1}^{2} b c h^{2} D 0 \\
+\frac{1}{4} b c h^{2}[4 D 20-2 C 0(1)-(s+t-u) D 1(1) \\
\left.-(s-t+u) D 1(2)+2 m_{\chi_{b}}^{2} D 1(3)\right] \\
\frac{16 \pi^{2} \Delta \mathcal{Q}_{L, 5,2}=-\frac{1}{16} h^{2}\left(\left(m_{\chi_{a}}+m_{\chi_{b}}\right) b c+m_{1} b d+m_{3} a c\right)[D 1(1)-D 1(2)]}{\Delta \mathcal{Q}_{L, 5,1}=\frac{1}{8} h^{2}\left(\left(m_{\chi_{a}}-m_{\chi_{b}}\right) b c-m_{1} b d+m_{3} a c\right)[D 1(1)-D 1(2)]} \\
\frac{16 \pi^{2}}{s} \Delta \mathcal{Q}_{L, 4,2}=-\frac{1}{4} b c h^{2}[D 1(1)-D 1(2)-D 2(3,1)+D 2(3,2)]
\end{gathered}
$$

\section{BOX PROTOTYPE 4b}

$$
\begin{aligned}
\frac{16 \pi^{2}}{s} \Delta \mathcal{Q}_{R, 1} & =\frac{1}{4} m_{\chi_{b}} a d f^{2}[D 1(2)-D 2(3,1)+2 D 2(3,2)-D 2(3,3)] \\
& +\frac{1}{4} m_{1} a c f^{2}[D 0-D 1(1)+2 D 1(2)-D 1(3)]
\end{aligned}
$$




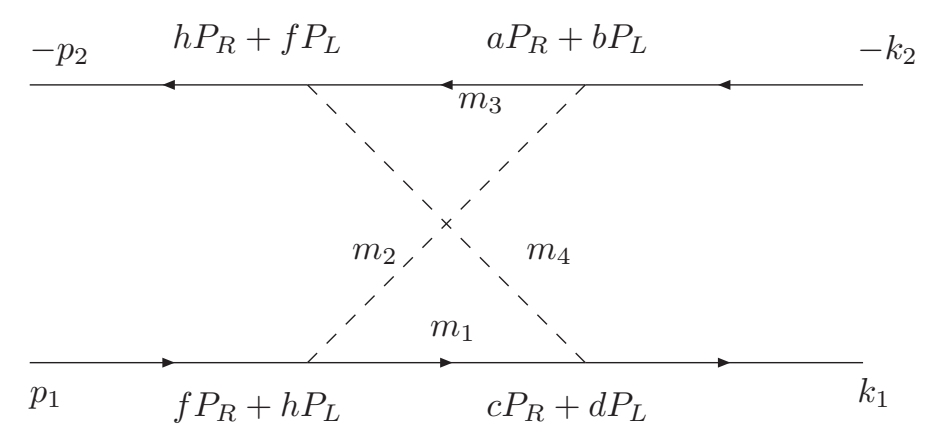

Figure 24: Box prototype graph 4b.

$$
\begin{aligned}
\frac{16 \pi^{2}}{s} \Delta \mathcal{Q}_{R, 2} & =\frac{1}{4} m_{\chi_{a}} a d f^{2}[D 1(1)+D 1(3)+D 2(3,1)+D 2(3,3)] \\
& -\frac{1}{4} m_{3} b d f^{2}[D 1(1)+D 1(3)]
\end{aligned}
$$

$$
\begin{aligned}
& \frac{16 \pi^{2}}{s} \Delta \mathcal{Q}_{R, 3,1}=\frac{1}{8} a d f^{2}\left(m_{\chi_{a}}^{2}-m_{\chi_{b}}^{2}\right)[D 1(1)-D 1(2)+D 1(3)]+\frac{1}{2} m_{1}^{2} a d f^{2} D 0 \\
&+\frac{1}{4} m_{\chi_{b}} m_{1} a c f^{2}[D 0-D 1(1)+2 D 1(2)-D 1(3)] \\
&-\frac{1}{4} m_{\chi_{a}} m_{3} b d f^{2}[D 1(1)+D 1(3)] \\
&-\frac{1}{8} a d f^{2}[8 D 20-4 C 0(1)+(s+t-3 u) D 1(1) \\
&-(s+t+u)(D 1(2)+D 1(3))] \\
& \frac{16 \pi^{2}}{s} \Delta \mathcal{Q}_{R, 3,2}=-\frac{1}{4} a d f^{2}[D 1(1)+D 2(3,1)-D 2(3,3)]
\end{aligned}
$$

$$
\begin{aligned}
& \frac{16 \pi^{2}}{s} \Delta \mathcal{Q}_{R, 4,1}=\frac{1}{4} m_{\chi_{a}} m_{\chi_{b}} a d f^{2}[D 1(1)-D 1(2)+D 1(3)]+\frac{1}{2} m_{1} m_{3} b c f^{2} D 0 \\
&-\frac{1}{4} m_{\chi_{a}} m_{1} a c f^{2}[D 0+D 1(1)+D 1(3)] \\
&-\frac{1}{4} m_{\chi_{b}} m_{3} b d f^{2}[D 1(1)-2 D 1(2)+D 1(3)] \\
& \frac{16 \pi^{2}}{s} \Delta \mathcal{Q}_{R, 4,2}=0
\end{aligned}
$$




$$
\begin{aligned}
& \frac{16 \pi^{2}}{s} \Delta \mathcal{Q}_{R, 5,1}=-\frac{1}{8} a d f^{2} m_{\chi_{b}}[D 1(1)-D 1(2)]+\frac{1}{8} a d f^{2} m_{\chi_{a}} D 1(3) \\
& +\frac{1}{8} m_{3} b d f^{2}[D 1(1)-D 1(3)]+\frac{1}{8} m_{1} a c f^{2}[D 0+D 1(1)-D 1(3)] \\
& \frac{16 \pi^{2}}{s} \Delta \mathcal{Q}_{R, 5,2}=\frac{1}{16} a d f^{2} m_{\chi_{b}}[D 1(1)-D 1(2)]+\frac{1}{16} a d f^{2} m_{\chi_{a}} D 1(3) \\
& +\frac{1}{16} m_{3} b d f^{2}[D 1(1)-D 1(3)]-\frac{1}{16} m_{1} a c f^{2}[D 0+D 1(1)-D 1(3)] \\
& \frac{16 \pi^{2}}{s} \Delta \mathcal{Q}_{L, 1}=\frac{1}{4} m_{\chi_{a}} b c h^{2}[D 1(1)+D 1(3)+D 2(3,1)+D 2(3,3)] \\
& -\frac{1}{4} m_{3} a c h^{2}[D 1(1)+D 1(3)] \\
& \frac{16 \pi^{2}}{s} \Delta \mathcal{Q}_{L, 2}=\frac{1}{4} m_{\chi_{b}} b c h^{2}[D 1(2)-D 2(3,1)+2 D 2(3,2)-D 2(3,3)] \\
& +\frac{1}{4} m_{1} b d h^{2}[D 0-D 1(1)+2 D 1(2)-D 1(3)] \\
& \frac{16 \pi^{2}}{s} \Delta \mathcal{Q}_{L, 3,1}=\frac{1}{4} m_{\chi_{a}} m_{\chi_{b}} b c h^{2}[D 1(1)-D 1(2)+D 1(3)]+\frac{1}{2} m_{1} m_{3} a d h^{2} D 0 \\
& -\frac{1}{4} m_{\chi_{a}} m_{1} b d h^{2}[D 0+D 1(1)+D 1(3)] \\
& -\frac{1}{4} m_{\chi_{b}} m_{3} a c h^{2}[D 1(1)-2 D 1(2)+D 1(3)] \\
& \frac{16 \pi^{2}}{s} \Delta \mathcal{Q}_{L, 3,2}=0 \\
& \frac{16 \pi^{2}}{s} \Delta \mathcal{Q}_{L, 4,1}=\frac{1}{8} b c h^{2}\left(m_{\chi_{a}}^{2}-m_{\chi_{b}}^{2}\right)[D 1(1)-D 1(2)+D 1(3)]+\frac{1}{2} m_{1}^{2} b c h^{2} D 0 \\
& +\frac{1}{4} m_{\chi_{b}} m_{1} b d h^{2}[D 0-D 1(1)+2 D 1(2)-D 1(3)] \\
& -\frac{1}{4} m_{\chi_{a}} m_{3} a c h^{2}[D 1(1)+D 1(3)] \\
& -\frac{1}{8} b c h^{2}[8 D 20-4 C 0(1)+(s+t-3 u) D 1(1) \\
& -(s+t+u)(D 1(2)+D 1(3))]
\end{aligned}
$$




$$
\begin{gathered}
\frac{16 \pi^{2}}{s} \Delta \mathcal{Q}_{L, 4,2}=-\frac{1}{4} b c h^{2}[D 1(1)+D 2(3,1)-D 2(3,3)] \\
\frac{16 \pi^{2}}{s} \Delta \mathcal{Q}_{L, 5,1}=-\frac{1}{8} b c h^{2} m_{\chi_{b}}[D 1(1)-D 1(2)]+\frac{1}{8} b c h^{2} m_{\chi_{a}} D 1(3) \\
+\frac{1}{8} m_{3} a c h^{2}[D 1(1)-D 1(3)]+\frac{1}{8} m_{1} b d h^{2}[D 0+D 1(1)-D 1(3)] \\
\frac{16 \pi^{2}}{s} \Delta \mathcal{Q}_{L, 5,2}=-\frac{1}{16} b c h^{2} m_{\chi_{b}}[D 1(1)-D 1(2)]-\frac{1}{16} b c h^{2} m_{\chi_{a}} D 1(3) \\
-\frac{1}{16} m_{3} a c h^{2}[D 1(1)-D 1(3)]+\frac{1}{16} m_{1} b d h^{2}[D 0+D 1(1)-D 1(3)]
\end{gathered}
$$

\section{BOX PROTOTYPE 5a}

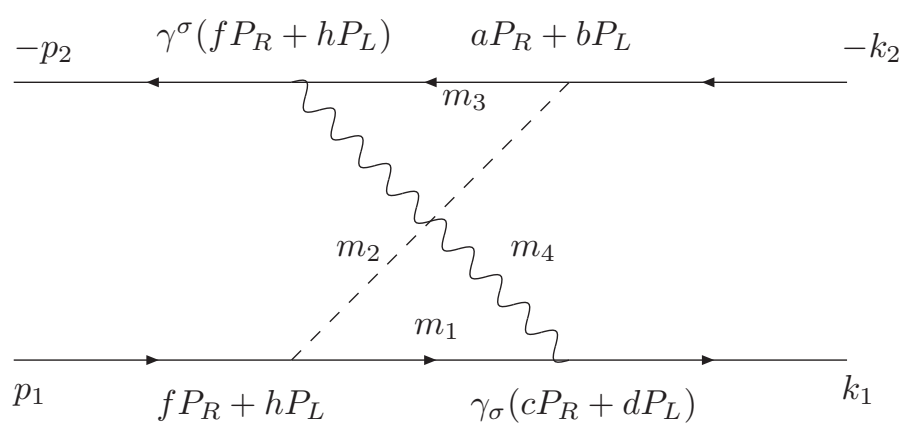

Figure 25: Box prototype graph 5a.

$$
\begin{gathered}
\frac{16 \pi^{2}}{s} \Delta \mathcal{Q}_{R, 1}=-\frac{1}{2} m_{\chi_{a}} b d f^{2}[D 1(2)+D 2(3,1)+D 2(3,3)] \\
+m_{3} a d f^{2} D 1(2) \\
\frac{16 \pi^{2}}{s} \Delta \mathcal{Q}_{R, 2}=-\frac{1}{2} m_{\chi_{b}} b d f^{2}[D 1(2)-D 2(3,1)+2 D 2(3,2)-D 2(3,3)] \\
+\frac{1}{2} m_{1} b c f^{2}[D 0-D 1(1)+2 D 1(2)-D 1(3)] \\
\frac{16 \pi^{2}}{s} \Delta \mathcal{Q}_{R, 3,1}=-\frac{1}{2} m_{\chi_{a}} m_{1} b c f^{2}[D 0+D 1(1)+D 1(3)]+m_{1} m_{3} a c f^{2} D 0
\end{gathered}
$$




$$
\frac{16 \pi^{2}}{s} \Delta \mathcal{Q}_{R, 3,2}=0
$$

$\frac{16 \pi^{2}}{s} \Delta \mathcal{Q}_{R, 4,1}=\frac{1}{2} m_{\chi_{b}} m_{1} b c f^{2}[D 0-D 1(1)+2 D 1(2)-D 1(3)]+2 b d f^{2} D 20$

$$
\frac{16 \pi^{2}}{s} \Delta \mathcal{Q}_{R, 4,2}=\frac{1}{2} b d f^{2}[D 1(2)+D 2(3,1)-D 2(3,3)]
$$

$$
\frac{16 \pi^{2}}{s} \Delta \mathcal{Q}_{R, 5,1}=\frac{1}{4} m_{1} b c f^{2}[D 0+D 1(1)-D 1(3)]
$$

$$
\frac{16 \pi^{2}}{s} \Delta \mathcal{Q}_{R, 5,2}=\frac{1}{8} m_{1} b c f^{2}[D 0+D 1(1)-D 1(3)]
$$

$$
\begin{aligned}
\frac{16 \pi^{2}}{s} \Delta \mathcal{Q}_{L, 1} & =-\frac{1}{2} m_{\chi_{b}} a c h^{2}[D 1(2)-D 2(3,1)+2 D 2(3,2)-D 2(3,3)] \\
& +\frac{1}{2} m_{1} a d h^{2}[D 0-D 1(1)+2 D 1(2)-D 1(3)]
\end{aligned}
$$

$$
\frac{16 \pi^{2}}{s} \Delta \mathcal{Q}_{L, 2}=-\frac{1}{2} m_{\chi_{a}} a c h^{2}[D 1(2)+D 2(3,1)+D 2(3,3)]+m_{3} b c h^{2} D 1(2)
$$

$$
\frac{16 \pi^{2}}{s} \Delta \mathcal{Q}_{L, 3,1}=\frac{1}{2} m_{\chi_{b}} m_{1} a d h^{2}[D 0-D 1(1)+2 D 1(2)-D 1(3)]+2 a c h^{2} D 20
$$

$$
\frac{16 \pi^{2}}{s} \Delta \mathcal{Q}_{L, 3,2}=\frac{1}{2} a c h^{2}[D 1(2)+D 2(3,1)-D 2(3,3)]
$$

$$
\begin{gathered}
\frac{16 \pi^{2}}{s} \Delta \mathcal{Q}_{L, 4,1}=-\frac{1}{2} m_{\chi_{a}} m_{1} a d h^{2}[D 0+D 1(1)+D 1(3)]+m_{1} m_{3} b d h^{2} D 0 \\
\frac{16 \pi^{2}}{s} \Delta \mathcal{Q}_{L, 4,2}=0 \\
\frac{16 \pi^{2}}{s} \Delta \mathcal{Q}_{L, 5,1}=\frac{1}{4} m_{1} a d h^{2}[D 0+D 1(1)-D 1(3)] \\
\frac{16 \pi^{2}}{s} \Delta \mathcal{Q}_{L, 5,2}=-\frac{1}{8} m_{1} a d h^{2}[D 0+D 1(1)-D 1(3)]
\end{gathered}
$$




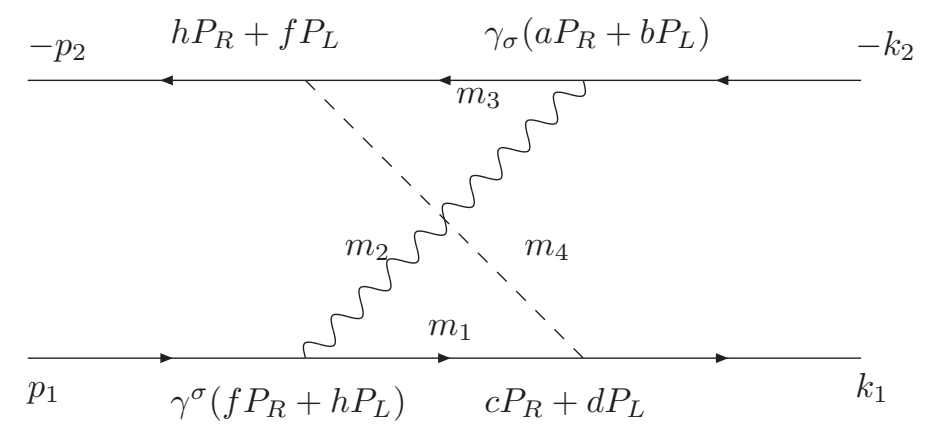

Figure 26: Box prototype graph 5b.

\section{BOX PROTOTYPE 5b}

$$
\begin{gathered}
\frac{16 \pi^{2}}{s} \Delta \mathcal{Q}_{R, 1}=-\frac{1}{2} m_{\chi_{a}} b c f^{2}[D 1(1)+D 1(3)+D 2(3,1)+D 2(3,3)] \\
-\frac{1}{2} m_{3} a c f^{2}[D 1(1)+D 1(3)] \\
\frac{16 \pi^{2}}{s} \Delta \mathcal{Q}_{R, 2}=\frac{1}{2} m_{\chi_{b}} b c f^{2}[D 1(1)-2 D 1(2)+D 1(3) \\
\quad+D 2(3,1)-2 D 2(3,2)+D 2(3,3)] \\
\quad-m_{1} b d f^{2}[D 0+D 1(2)] \\
\frac{16 \pi^{2}}{s} \Delta \mathcal{Q}_{R, 3,1}=-\frac{1}{2} m_{\chi_{b}} m_{3} a c f^{2}[D 1(1)-2 D 1(2)+D 1(3)]+m_{1} m_{3} a d f^{2} D 0 \\
\quad \frac{16 \pi^{2}}{s} \Delta \mathcal{Q}_{R, 3,2}=0 \\
\frac{16 \pi^{2}}{s} \Delta \mathcal{Q}_{R, 4,1}=-\frac{1}{2} m_{\chi_{a}} m_{3} a c f^{2}[D 1(1)+D 1(3)]+2 b c f^{2} D 20 \\
\frac{16 \pi^{2}}{s} \Delta \mathcal{Q}_{R, 4,2}=\frac{1}{2} b c f^{2}[D 1(1)-D 1(3)+D 2(3,1)-D 2(3,3)] \\
\frac{16 \pi^{2}}{s} \Delta \mathcal{Q}_{R, 5,1}=\frac{1}{4} m_{3} a c f^{2}[D 1(1)-D 1(3)]
\end{gathered}
$$




$$
\frac{16 \pi^{2}}{s} \Delta \mathcal{Q}_{R, 5,2}=-\frac{1}{8} m_{3} a c f^{2}[D 1(1)-D 1(3)]
$$

$$
\begin{aligned}
\frac{16 \pi^{2}}{s} \Delta \mathcal{Q}_{L, 1} & =\frac{1}{2} m_{\chi_{b}} a d h^{2}[D 1(1)-2 D 1(2)+D 1(3)+D 2(3,1)-2 D 2(3,2)+D 2(3,3)] \\
& -m_{1} a c h^{2}[D 0+D 1(2)]
\end{aligned}
$$

$$
\begin{aligned}
\frac{16 \pi^{2}}{s} \Delta \mathcal{Q}_{L, 2} & =-\frac{1}{2} m_{\chi_{a}} a d h^{2}[D 1(1)+D 1(3)+D 2(3,1)+D 2(3,3)] \\
& -\frac{1}{2} m_{3} b d h^{2}[D 1(1)+D 1(3)]
\end{aligned}
$$

$$
\frac{16 \pi^{2}}{s} \Delta \mathcal{Q}_{L, 3,1}=-\frac{1}{2} m_{\chi_{a}} m_{3} b d h^{2}[D 1(1)+D 1(3)]+2 a d h^{2} D 20
$$

$$
\frac{16 \pi^{2}}{s} \Delta \mathcal{Q}_{L, 3,2}=\frac{1}{2} a d h^{2}[D 1(1)-D 1(3)+D 2(3,1)-D 2(3,3)]
$$

$$
\frac{16 \pi^{2}}{s} \Delta \mathcal{Q}_{L, 4,1}=-\frac{1}{2} m_{\chi_{b}} m_{3} b d h^{2}[D 1(1)-2 D 1(2)+D 1(3)]+m_{1} m_{3} b c h^{2} D 0
$$

$$
\frac{16 \pi^{2}}{s} \Delta \mathcal{Q}_{L, 4,2}=0
$$

$$
\begin{aligned}
& \frac{16 \pi^{2}}{s} \Delta \mathcal{Q}_{L, 5,1}=\frac{1}{4} m_{3} b d h^{2}[D 1(1)-D 1(3)] \\
& \frac{16 \pi^{2}}{s} \Delta \mathcal{Q}_{L, 5,2}=\frac{1}{8} m_{3} b d h^{2}[D 1(1)-D 1(3)]
\end{aligned}
$$

All other box diagram prototypes that give contributions to these amplitudes can be obtained from the above box prototypes by means of crossing relations (e.g. $u \leftrightarrow t$, and $\left.m_{\chi_{a}} \leftrightarrow m_{\chi_{b}}\right)$. 


\section{References}

[1] J. Ellis, G. Ganis, D.V. Nanopoulos and K.A. Olive, hep-ph/0009355

[2] G.L. Kane, S.F. King and L.-T. Wang hep-ph/0010312

[3] J. Ellis and D.A. Ross hep-ph/0012067

[4] S.Y. Choi, A. Djouadi, M. Guchait, J. Kalinowski, H.S. Song and P.M. Zerwas Eur. Phys. J. C14 (2000) 535;

S.Y. Choi, A. Djouadi, H. Dreiner, J. Kalinowski, and P.M. Zerwas Eur. Phys. J. C7 (1999)123;

J.Kalinowski hep-ph/9905558

[5] M. A. Diaz, S. F. King, D. A. Ross Nucl. Phys. B 529 (1998) 23

[6] S. Kiyoura, M. M. Nojiri, D. M. Pierce and Y. Yamada, Phys. Rev. D 58 (1998) 075002;

M. A. Diaz, S. F. King and D. A. Ross hep-ph/0008117

[7] T. Blank and W. Hollik, hep-ph/0011092

[8] G. Passarino and M. Veltman, Nucl. Phys. B 160 (1979) 151

[9] D.M. Pierce, J.A.Bagger, K.Matchev and R. Zhang, Nucl. Phys. B B491 (1997) 3

[10] G.J. van Oldenborgh, Comput. Phys. Commun. 66 (1991) 1

[11] J.A. M. Vermaseren, math-ph/0010025 